\title{
CT10 next-to-next-to-leading order global analysis of QCD
}

\author{
Jun Gao, ${ }^{1}$ Marco Guzzi, ${ }^{2}$ Joey Huston, ${ }^{3}$ Hung-Liang Lai, ${ }^{4}$ Zhao Li, ${ }^{5}$ Pavel Nadolsky, ${ }^{1}$ Jon Pumplin, ${ }^{3}$ \\ Daniel Stump, ${ }^{3}$ and C.-P. Yuan ${ }^{3,6}$ \\ ${ }^{1}$ Department of Physics, Southern Methodist University, Dallas, Texas 75275-0181, USA \\ ${ }^{2}$ Deutsches Elektronensynchrotron DESY, Notkestrasse 85, D-22607 Hamburg, Germany \\ ${ }^{3}$ Department of Physics and Astronomy, Michigan State University, \\ East Lansing, Michigan 48824-1116, USA \\ ${ }^{4}$ Taipei Municipal University of Education, Taipei 10048, Taiwan \\ ${ }^{5}$ Institute of High Energy Physics, Chinese Academy of Sciences, Beijing 100049, China \\ ${ }^{6}$ Center for High Energy Physics, Peking University, Beijing 100871, China
}

(Received 20 March 2013; published 19 February 2014)

\begin{abstract}
We present next-to-next-to-leading order (NNLO) parton distribution functions (PDFs) from the CTEQTEA group. The CT10NNLO PDF fit is based on essentially the same global data sets used in the CT10 and CT10W NLO PDF analyses. After exploring the goodness of the fits to the HERA combined data and the Tevatron jet data, we present various predictions at NNLO accuracy for both existing and forthcoming precision measurements from the CERN Large Hadron Collider. The range of variations in the gluon distribution introduced by correlated systematic effects in inclusive jet production is also examined.
\end{abstract}

DOI: 10.1103/PhysRevD.89.033009

PACS numbers: 12.15.Ji, 12.38.Cy, 13.85.Qk

\section{INTRODUCTION}

A global analysis of perturbative QCD makes use of experimental data from many short-distance scattering processes to construct, within some approximation, universal parton distribution functions (PDFs) for the proton. These PDFs can be used to calculate hadronic cross sections. The CTEQ global analysis at the next-to-leading order (NLO) in the strong coupling constant $\alpha_{s}$ has been developed over decades. Examples of general-purpose PDFs in this series include CTEQ6, published in 2002 [1], followed by CTEQ6.1 in 2003 [2]. Starting from CTEQ6.5 published in 2006 [3], and in ensuing PDF sets such as CTEQ6.6 [4] and CT09 [5], the effects of finite quark masses on the CTEQ global analysis have been implemented in the S-ACOT- $\chi$ factorization scheme [6-9] at NLO accuracy. The most recent CTEQ NLO PDFs, named CT10 and CT10W, were published in 2010 [10] and are currently in wide use in phenomenological predictions for the Tevatron, LHC, and other experiments.

The data from the CERN Large Hadron Collider (LHC) cover a wide kinematic range with high expected precision; a similar level of precision is needed for the theoretical predictions. Thus, there is a need for using cross sections calculated up to next-to-next-to-leading order (NNLO) in the strong coupling constant $\alpha_{s}$ with parton distribution function sets that are also determined at NNLO. Complete calculations for this order in $\alpha_{s}$ are available for the running coupling $\alpha_{\mathrm{s}}(Q)$, PDF evolution in $Q[11,12]$, matrix elements in deepinelastic scattering (DIS) [13-19], and vector boson production [20,21]. NNLO matrix elements are unknown for several other processes in the global analysis. Most notably, the key input theoretical cross section for inclusive jet production in $p \stackrel{(-)}{p}$ collisions is still evaluated at NLO, although some of the NNLO radiative contributions have already been computed $[22,23] .{ }^{1}$ Various NNLO PDF sets have been published in the literature [24-29] that make use of NNLO matrix elements when available.

In this paper, we present a new generation of CTEQ parton distributions, at NNLO, named CT10 NNLO ${ }^{2}$ They are obtained from a global analysis of QCD data in which three changes have been made to include the calculations at NNLO. First, the parton distribution functions $f(x, Q)$ are evolved according to the 3-loop Dokshitzer-GribovLipatov-Altarelli-Parisi (DGLAP) equations. Second, the strong coupling $\alpha_{\mathrm{s}}\left(\mu_{R}\right)$ evolves in the renormalization scale $\mu_{R}$ according to the 3-loop beta function. Third, the hard matrix elements for DIS and vector boson production are calculated up to two QCD loops. A detailed implementation of neutral-current DIS cross sections in the S-ACOT- $\chi$ scheme, which performs a consistent treatment of nonzero masses of heavy partons up to this order of accuracy, is documented in Ref. [30] and recapped in Sec. II.

The experimental data sets included in the CT10NNLO fit are essentially the same as in the CT10 and CT10W NLO fits, with the exceptions of Tevatron Run-1 inclusive jet data and a subset of the Tevatron Run-2 lepton charged asymmetry data from $W$ boson decays. The changes in the selection of the experimental data sets are summarized in Sec. IV. No LHC data are included in the CT10 NNLO

\footnotetext{
${ }^{1}$ Some other cross sections, such as for Higgs boson production and diboson production, have been calculated to NNLO, but to date have not been implemented in any global analysis.

${ }^{2}$ Parametrizations of the CT10NNLO PDFs were publicly released in 2012 [31].
} 
analysis, which can therefore be used to make predictions based exclusively on the pre-LHC data. (The impact of the HERA and LHC data published after 2010 will be investigated in the post-CT10 fits.)

Since the CTEQ4 analysis released in 1996 [32,33], hadron-hadron collider measurements on the production of hadronic jets are included in CTEQ fits to provide pivotal information about the high- $x$ gluon distribution. To achieve good agreement with Tevatron inclusive jet cross sections, it is generally necessary to assume a larger gluon PDF that would be preferred solely on the basis of DIS experiments; and the uncertainty in the gluon PDF at $x>0.1$ is reduced dramatically in the global fits that utilize the collider jet data than in the fits without them [5].

The inclusive jet cross sections play as a prominent role in the CT10 NNLO analysis; however, as stated previously, they are still evaluated with NLO matrix elements, and hence require special scrutiny. The experimental data on the inclusive jet cross sections are statistically very precise but have significant systematic uncertainties. The partial NNLO contributions to the LHC jet production reach 10\%-20\% [23], and their magnitude is about the same as those of the experimental systematic effects, and possibly indistinguishable from the latter. We examine variations in the gluon and other PDFs caused by the QCD scale dependence and by various treatments of systematic uncertainties in inclusive jet production. The impact of jet-related uncertainties is compared to those from other sources.

For consistency with the CT10 NLO PDFs, CT10 NNLO assumes the same value of the QCD coupling strength $\alpha_{s}\left(M_{Z}\right)=0.118$ and pole masses for heavy quarks of $m_{c}=1.3 \mathrm{GeV}$ and $m_{b}=4.75 \mathrm{GeV}$. A commentary on the choice of the heavy-quark masses and a description of heavy-quark production in DIS is presented in Sec. IIC. The CT10NNLO eigenvector PDF sets are available on the CTEQ Web site [34] and in the LHAPDF standard format [35]. Together with the PDF eigenvector sets for the central value of $\alpha_{s}\left(M_{Z}\right)=0.118$, an additional PDF series in which $\alpha_{s}\left(M_{Z}\right)$ is varied in the range 0.112-0.127 are provided. These PDFs are sufficient for computing the correlated $\mathrm{PDF}+\alpha_{s}$ uncertainty by adding the $\mathrm{PDF}$ and $\alpha_{s}$ uncertainties in quadrature, as explained in Ref. [36].

The outline of the paper is as follows. Section II reviews our NNLO implementation of DIS cross sections, placing an emphasis on the treatment of heavy-quark contributions. Section III summarizes the statistical procedure of the global analysis, most notably the definition of the loglikelihood function and the implementation of correlated systematic errors. Section IV lists the experimental data used in the CT10NNLO analysis. Section V describes some features of the resulting CT10NNLO PDFs, while Section VI presents detailed comparisons of data and theory. Section VII presents NNLO predictions for collider measurements based on the CT10NNLO PDFs, and Section VIII contains our summary and conclusions.

\section{HEAVY-FLAVOR SCHEME IN THE CT10NNLO FIT}

\section{A. QCD factorization for heavy quarks in the S-ACOT- $\chi$ scheme}

A consistent implementation of contributions from the massive quarks ( $c$ and $b$ ) is a prerequisite and challenge for a viable NNLO PDF analysis. The mass dependence of the heavy-quark DIS contributions to PDF fits affects QCD precision observables in a wide range of energies [3]. Heavy-quark mass effects were studied in PDF fits since the mid-1990s in the context of several theoretical approaches, or "heavy-quark schemes." The S-ACOT- $\chi$ factorization scheme [6-9] has been adopted in the recent NLO fits CTEQ6HQ [37], 6.5 [3], 6.6 [4], and CT10 [10]. For the present work, the S-ACOT- $\chi$ scheme has been extended to NNLO accuracy, i.e., $\mathcal{O}\left(\alpha_{s}^{2}\right)$, in the computation of neutral-current DIS cross sections [30]. ${ }^{3}$ The alternative $\mathrm{TR}^{\prime}$ scheme $[38,39]$ is used in the MSTW and HERAPDF fits, while the FONLL scheme [40,41] has been adopted by the NNPDF Collaboration. The BMSN scheme [42-44] and the fixed-flavor number (FFN) schemes are used by the $\mathrm{ABM}$ and GJR groups, respectively.

The general-mass variable flavor number (GM-VFN) schemes replace the zero-mass approximation, which is no longer adequate for describing the DIS data. They evaluate the coefficient functions using the exact dependence on heavy-quark mass $m_{h}$, while heavy-quark PDFs provide an approximation for collinear production of $h \bar{h}$ pairs when $Q^{2} \gg m_{h}^{2}$. They are valid across the whole range of $Q$ values accessed in the global fits. ${ }^{4}$

The heavy-quark schemes are brought into better consistency among themselves when going from NLO to NNLO calculations [30,47]. For example, the spread of theoretical predictions for the standard-candle $W$ and $Z$ boson production cross sections at the LHC has shrunk from $6 \%$ to $8 \%$ at NLO [4] (with the largest deviations observed with the zero-mass PDFs) to less than $3 \%$ at NNLO [48-53]. Quark-mass effects continue to be important. Their nonequivalent treatment by various PDF analysis groups gives rise to residual uncertainties in the standard candle predictions and in the observables sensitive to the gluon or heavy-quark scattering, such as Higgs boson production.

The NNLO calculation in the S-ACOT- $\chi$ scheme in Ref. [30] focused on two issues that had not been earlier addressed. First, we clarified the connection of

\footnotetext{
${ }^{3}$ The charged-current DIS cross sections, for which some $\mathcal{O}\left(\alpha_{s}^{2}\right)$ massive amplitudes are not available, are evaluated at NLO.

${ }^{4} \mathrm{~A}$ complementary approach (an intermediate-mass scheme) uses approximate quark mass dependence in all scattering channels [45]. DIS cross sections in this approach utilizing the exact $O\left(\alpha_{s}\right)$ massive ACOT terms and approximate $O\left(\alpha_{s}^{2}\right)$ and $O\left(\alpha_{s}^{3}\right)$ massive terms have been recently published [46].
} 
the GM-VFN scheme at NNLO to the proof of QCD factorization for DIS with massive quarks presented by Collins [7]. It was demonstrated that the S-ACOT- $\chi$ scheme can be derived to all orders from Collins's approach and is validated by a QCD factorization theorem.

Second, we documented an algorithm that organizes the NNLO S-ACOT- $\chi$ calculation in close analogy to the zeromass VFN computation. As a result, the NNLO DIS cross sections can be constructed step-by-step from the components that can be found in literature. In the S-ACOT- $\chi$ scheme, all elements arise from the all-order factorization formalism, which is not always the case in other frameworks. These features distinguish the S-ACOT- $\chi$ scheme from the $\mathrm{TR}^{\prime}$ and FONLL schemes that follow different implementation approaches.

As a result, the structure of the S-ACOT- $\chi$ cross sections is readily reproducible; the universality of the PDFs follows from the Collins proof of QCD factorization [30]. The $Q$ dependence of the QCD coupling and PDFs is found by numerical QCD evolution that assumes one shared $N_{f}$ value in each $Q$ range; QCD quantities for $N_{f}$ and $N_{f}+1$ active flavors are related at the switching energy scales through the matching conditions. The NNLO radiative contributions are assembled straightforwardly from the matrix elements in the massive FFN [17-19] and zeromass VFN schemes [13-16], as well as from the massdependent operator matrix elements for the heavy-quark PDFs [42].

The S-ACOT- $\chi$ scheme reduces to the FFN scheme at the heavy-quark threshold $Q^{2} \approx m_{h}^{2}$ and to the zero-mass $\overline{\mathrm{MS}}$ scheme at $Q^{2} \gg m_{h}^{2}$, without additional renormalization. The matching of the GM-VFN cross sections to the FFN cross sections near the mass threshold is generally not automatic. To realize it, the $\mathrm{TR}^{\prime}$ and FONLL scheme introduce additional elements (constraints on the $Q$ dependence of the heavy-quark DIS contributions in the $\mathrm{TR}^{\prime}$ scheme and the "damping factor" in the FONLL framework) that are not stipulated by the QCD factorization theorem. In the S-ACOT- $\chi$ scheme, threshold matching is rather a consequence of the energy conservation condition that suppresses the difference between the GM-VFN and FFN results when $Q^{2}$ approaches $m_{h}^{2}$. This is achieved by restricting the allowed form for the approximate coefficient functions that describe QCD scattering off an initial-state heavy quark, so that they comply with energy conservation. It leads to the effective rescaling ( $\chi$ rescaling) of the lightcone momentum fraction in the approximate heavy-quark scattering terms $[9,54]$.

The derivation of mass-dependent contributions from the QCD factorization theorem, which is shown to be compatible with the rescaled terms, leads to more confident constraints on the PDFs. Our fitting code implements these NNLO Wilson coefficient functions in the S-ACOT- $\chi$ scheme together with the HOPPET program for the evolution of $\alpha_{s}$ and PDFs [55], in which the switching points between the active flavors can be expressed in terms of either the $\overline{\mathrm{MS}}$ masses or the pole masses. The fitting program can read either the pole masses or the $\overline{\mathrm{MS}}$ masses as an input.

In the latter case, the $\overline{\mathrm{MS}}$ masses are converted into the pole masses when needed, e.g., to evaluate those operator matrix elements $A_{a b}^{(2)}$ that are published in terms of the pole masses. Although the $\overline{\mathrm{MS}}$ masses are known more precisely than the pole masses, using the $\overline{\mathrm{MS}}$ mass at the NNLO level does not lead to a more accurate fit, as the $\overline{\mathrm{MS}} \rightarrow$ pole conversion in the DIS cross sections introduces an additional perturbative uncertainty that overrides the precision of the $\overline{\mathrm{MS}}$ input. In the CT10 NNLO fit, the pole masses $m_{c}=1.3 \mathrm{GeV}$ and $m_{b}=4.75 \mathrm{GeV}$ have been assumed. Complementary fits in which the $\overline{\mathrm{MS}}$ charm mass was chosen as the input and constrained by the global data were also carried out [53].

\section{B. The S-ACOT- $\chi$ scheme at NNLO in a nutshell}

In the S-ACOT- $\chi$ scheme, a generic inclusive structure function $F_{2, L} \equiv F$ takes the form of a convolution product $(\otimes)$ of the Wilson coefficient functions $C_{i a}$ and the parton distribution functions $f_{a / p}(\xi, \mu)$,

$$
\begin{aligned}
F(x, Q) & =\sum_{i=1}^{N_{f}^{f s}} e_{i}^{2} \sum_{a=0}^{N_{f}} \int_{x}^{1} \frac{d \xi}{\xi} C_{i a}\left(\frac{x}{\xi}, \frac{Q}{\mu}, \frac{m_{h}}{\mu}, \alpha_{s}(\mu)\right) f_{a / p}(\xi, \mu) \\
& \equiv \sum_{i=1}^{N_{f}^{f s}} e_{i}^{2} \sum_{a=0}^{N_{f}}\left[C_{i a} \otimes f_{a / p}\right](x, Q)
\end{aligned}
$$

where $\xi$ is the light-cone momentum fraction, $\mu$ is the factorization scale, $N_{f}$ indicates the number of active flavors, and $N_{f}^{f s}$ is the number of the produced final-state flavors (most generally, $N_{f}^{f s} \neq N_{f}$ ). The structure function can also be written as

$$
F=\sum_{l=1}^{N_{l}} F_{l}+\sum_{h=N_{l}+1}^{N_{f}^{f_{s}}} F_{h},
$$

where $l$ and $h$ are the indices of light-quark and heavy-quark flavors probed by the photon, respectively [41]. (Note that the S-ACOT- $\chi$ scheme can simultaneously account for several flavors with nonzero masses.) On the right-hand side,

$$
\begin{aligned}
F_{l} & =e_{l}^{2} \sum_{a}\left[C_{l, a} \otimes f_{a / p}\right](x, Q), \\
F_{h} & =e_{h}^{2} \sum_{a}\left[C_{h, a} \otimes f_{a / p}\right](x, Q) .
\end{aligned}
$$

The $\mathcal{O}\left(\alpha_{s}^{2}\right)$ radiative contributions, $F_{l}^{(2)}$ and $F_{h}^{(2)}$, are 


$$
\begin{gathered}
F_{l}^{(2)}=e_{l}^{2}\left\{C_{l, l}^{N S,(2)} \otimes\left(f_{l / p}+f_{\bar{l} / p}\right)\right. \\
\left.+c^{P S,(2)} \otimes \Sigma+c_{l, g}^{(2)} \otimes f_{g / p}\right\}, \\
F_{h}^{(2)}=e_{h}^{2}\left\{c_{h, h}^{N S,(2)} \otimes\left(f_{h / p}+f_{\bar{h} / p}\right)+C_{h, l}^{(2)} \otimes \Sigma\right. \\
\left.+C_{h, g}^{(2)} \otimes f_{g / p}\right\},
\end{gathered}
$$

where the lowercase notation $c_{a, b}^{(2)}$ indicates a zero-mass Wilson coefficient function, the uppercase notation $C_{a, b}^{(2)}$ indicates a massive coefficient function, and $\Sigma(x, \mu)=$ $\sum_{i=1}^{N_{f}}\left[f_{i / p}(x, \mu)+f_{\bar{i} / p}(x, \mu)\right]$ denotes the singlet-quark PDF. These equations have the same form as the factorized expressions for the zero-mass structure functions. Their components are listed explicitly in Ref. [30]. In this derivation, we employ a rescaling convention [9] to construct the LO, NLO, and NNLO coefficient functions with initial-state heavy quarks, $c_{h, h}^{(k)}$ with $k=0,1$, and 2 , and the associated subtraction terms. They are obtained by evaluating the zero-mass expressions as a function of the rescaling variable $\chi$,

$$
c_{h, h}^{(k)}\left(\frac{x}{\xi}, \frac{Q}{\mu}, \frac{m_{h}}{Q}\right)=c_{h, h}^{(k)}\left(\frac{\chi}{\xi}, \frac{Q}{\mu}, m_{h}=0\right) \theta(\chi \leq \xi \leq 1),
$$

where

$$
\chi=x\left(1+\frac{\left(\sum_{f s} m_{h}\right)^{2}}{Q^{2}}\right),
$$

and $\sum_{f s} m_{h}$ is the sum of the heavy-quark masses in the final state (equal to $2 m_{h}$ in the lowest-order $c \bar{c}$ pair production). These rescaled coefficient functions obey energy conservation and vanish near the production threshold, so that the FFN result is reproduced in this limit.

Equations (4) and (5) apply to the inclusive DIS functions $F_{2}, F_{L}$, etc. In the case of semi-inclusive DIS production of heavy quarks, the definition of the semiinclusive (SI) structure functions $F_{h, \mathrm{SI}}$, such as $F_{2}^{c \bar{c}}$ measured at HERA, requires additional care in order to obtain infrared-safe results at all $Q$ [43]. In the CT10NNLO global fit, the following approximation for $F_{h, \mathrm{SI}}$ has been adopted $[30,41]$. At moderate $Q$ values accessible at HERA, it is defined as

$$
F_{h, \mathrm{SI}}^{(2)}(x, Q)=F_{h}^{(2)}(x, Q)+\sum_{l=1}^{N_{l}} e_{l}^{2} L_{I, q}^{N S,(2)} \otimes\left(f_{l / p}+f_{\bar{l} / p}\right),
$$

where $F_{h}^{(2)}(x, Q)$ is the $\mathcal{O}\left(\alpha_{s}^{2}\right)$ contribution to the inclusive DIS function $F(x, Q)$ arising from photon scattering on a heavy quark; cf. Eq. (5). The function $L_{I, q}^{N S,(2)}(\xi, Q / \mu$, $\left.m_{h} / \mu\right)$ is the nonsinglet part of the light-quark component $F_{l}(x, Q)$ that contains radiation of a $h \bar{h}$ pair in the final state, computed in Ref. [19]. In the kinematic region $Q<10 \mathrm{GeV}$, which supports most of the HERA charm production data, we observe that $L_{I, q}^{N S,(2)}$ contributes about $0 \%-3 \%$ on the semi-inclusive charm cross section; i.e., it is small compared to the typical experimental errors.

\section{A phenomenological illustration}

In Fig. 1, the CT10 NLO/NNLO predictions for $F_{2}^{c \bar{c}}$ are compared to a recent data set from the H1 Collaboration [56], in which the charm structure function $F_{2}^{c \bar{c}}$ was extracted from the $D^{*}$ meson distribution. Predictions for $F_{2}^{c \bar{c}}$ are computed as a function of the momentum fraction $x$ for five different bins of $Q$. The dashed red lines and solid blue lines represent the S-ACOT- $\chi$ predictions at NLO and NNLO, respectively. Although this specific data set [56] was not included in the CT10 fits, the overall agreement is very good. The NLO and NNLO predictions are close to

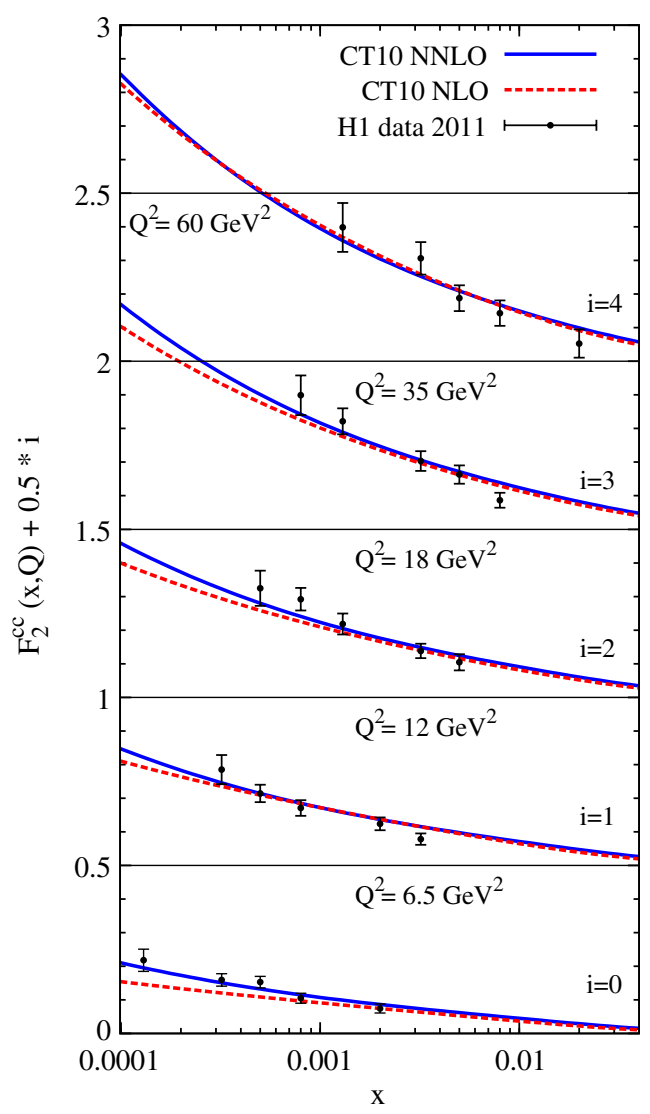

FIG. 1 (color online). The semi-inclusive heavy-quark function $F_{2}^{c \bar{c}}$ as a function of $x$ in different bins of $Q$. The CT10NLO, CT10NNLO predictions and H1 data [56] are compared. 
one another for the most part, at least in the intermediate $10^{-3} \leq x \leq 10^{-2}$ kinematic region, in which the bulk of the data was collected. However, the NLO prediction is shown for a particular factorization scale that improved agreement with the data, $\mu=\sqrt{Q^{2}+m_{c}^{2}}$, while a more typical scale choice $\mu=Q$ was taken at NNLO. In general, the NLO predictions for $F_{2}^{c \bar{c}}$ are characterized by a wide band of the scale dependence uncertainty. This band is reduced significantly when going to NNLO, and the NNLO predictions in the S-ACOT- $\chi$ scheme are closer to those in the FONLL-C and $\mathrm{TR}^{\prime}$ schemes [30]. The NNLO predictions are therefore more robust compared to NLO.

Soon after the CT10 NNLO PDFs were released, several DIS charm production cross section measurements by $\mathrm{H} 1$ and ZEUS that we use [57-60] were combined into one set [61]. We compared NNLO fits to the separate and combined HERA charm data sets. Their resulting PDFs and uncertainties turned out to be very similar [53]. The combined HERA charm data agrees well with the CT10 NNLO prediction: $\chi^{2} / N_{p t}=55.4 / 47=1.18$ for the default charm pole mass $m_{c}^{\text {pole }}=1.3 \mathrm{GeV}$ that we use.

The pole mass of $1.3 \mathrm{GeV}$ is compatible with the preferred $\overline{\mathrm{MS}}$ charm mass $m_{c}\left(m_{c}\right)$ determined from a simultaneous fit of PDFs and $m_{c}\left(m_{c}\right)$ in [53]. In that paper, the figure-of-merit function $\chi^{2}$ for the global hadronic data (including the combined HERA charm data set) was examined as a function of $m_{c}\left(m_{c}\right)$.

The preferred value was determined to be $m_{c}\left(m_{c}\right)=$ $1.19_{-0.15}^{+0.08} \mathrm{GeV}$ at $68 \%$ C.L., where the error is a quadrature sum of PDF and theoretical uncertainties. ${ }^{5}$ This value, constrained primarily by a combination of inclusive and charm production measurements in HERA deepinelastic scattering, translates into $m_{c}^{\text {pole }}=1.31_{-0.16}^{+0.09} \mathrm{GeV}$ and $1.58_{-0.15}^{+0.08} \mathrm{GeV}$ if using the conversion formula in Eq. (17) of [62] at one and two loops with $\alpha_{s}\left(M_{Z}, N_{f}=5\right)=0.118$. Either converted value is compatible at $2 \sigma$ with $m_{c}^{\text {pole }}=1.3 \mathrm{GeV}$ assumed by CT10 NNLO.

It is also interesting to note that this best-fit $m_{c}\left(m_{c}\right)$ agrees with the outcome of an independent PDF fit in the FFN scheme at the same order in $\alpha_{s}$ (at two loops), $m_{c}\left(m_{c}\right)=1.15 \pm 0.04(\exp )_{-0.00}^{+0.04}$ (scale) $\mathrm{GeV}[63]{ }^{6}$ In both the S-ACOT- $\chi$ and FFN fits, the central $m_{c}\left(m_{c}\right)$ values are lower than the world-average $1.275 \pm$ $0.025 \mathrm{GeV}$ [64], but the remaining difference disappears upon considering variations due to the choice of the rescaling variable in the S-ACOT- $\chi$ scheme (quantifying to some extent the missing higher-order contributions) or

\footnotetext{
${ }^{5}$ In contrast, the fit is hardly sensitive to the bottom quark mass, given the smallness of bottom-scattering contributions to the fitted cross sections.

${ }^{6}$ The disparate magnitudes of the uncertainties on $m_{c}\left(m_{c}\right)$ in two fits are caused mostly by their different definitions of the PDF uncertainties adopted by the ABM and CT10 groups.
}

including the approximate three-loop contribution to massive quark DIS in the FFN scheme [63].

The best-fit $m_{c}\left(m_{c}\right)$ and the PDFs that come with it are sensitive to the implementation of heavy-quark terms, such as the truncation of the perturbative conversion of the $\overline{\mathrm{MS}}$ to the pole mass, $\lambda$ parameter in the rescaling correction, and implementation of correlated effects for the combined (charm) HERA data. The associated errors in the key LHC cross sections have been examined and found to be mild and to lie within the usual $90 \%$ C.L. PDF uncertainty [53]. We do not separate them from the PDF uncertainty, which has a comparable uncertainty of its own. The corresponding variations in the NNLO $W$ or $Z$ cross section at the LHC are below $2 \%$, i.e. reduced comparatively to the uncertainties due the heavy-quark scheme observed previously at NLO [4].

\section{GLOBAL ANALYSIS WITH CORRELATED SYSTEMATIC ERRORS}

For completeness, and to establish notations for the ensuing discussion, in this section we summarize the statistical procedure adopted in the CT10NNLO analysis. An important aspect of this procedure is to determine the uncertainties of the PDFs, which arise from several sources, both experimental and theoretical.

Experimental uncertainties may be uncorrelated between different measurements, such as the bin-by-bin statistical error on the measured cross section. Some experimental uncertainties are highly correlated between different measurements within one experiment or even among several experiments. The luminosity error is an example of a correlated experimental uncertainty in a data set. It affects equally the normalization of all cross section measurements from the experiment. Other correlated uncertainties typically exist and may have an impact that is comparable to the luminosity error.

Inclusion of the correlated experimental errors utilizing their standard deviations published by the experiments began in the CTEQ program with the construction of the CTEQ6 parton distributions [1]. This is achieved by constructing an appropriate figure-of-merit function $\chi^{2}$ that includes errors from both uncorrelated and correlated sources, as shown explicitly in Sec. IIIA.

By examining the $\chi^{2}$ function in the neighborhood of the best fit and its dependence on $\alpha_{s}$, the CT10 global analysis determines a family of independent eigenvector sets that can be used to propagate the combined $\mathrm{PDF}+\alpha_{s}$ uncertainty into theoretical predictions (cf. Sec. IIIB). The central PDF set and PDF eigenvector sets are generally sensitive to the implementation of correlated systematic errors in the fit. Possible procedures for reconstructing the correlation error matrix from the published standard deviations, and the expected modifications in the PDFs that they induce, are discussed in Sec. IIIC. A numerical comparison of these procedures will be presented in Sec. VI. 


\section{A. Log-likelihood function in the CT10 NNLO analysis}

A typical experiment $E$ in the global fit publishes a set of measurements $\left\{M_{i} ; i=1,2,3, \ldots, N_{p t}\right\}$, consisting of a central value $D_{i}$ for the observable, a standard deviation for the uncorrelated experimental error $s_{i}$, and standard deviations $\beta_{k \alpha}$ for each of $N_{\lambda}$ systematic errors, where $k=1,2,3, \ldots, N_{\lambda}$. We do not know the experimental errors, but experiments provide their standard deviations. So we write

$$
D_{i}=X_{i}+s_{i} \delta_{i}+\sum_{\alpha=1}^{N_{\lambda}} \beta_{k \alpha} \lambda_{\alpha}
$$

where $X_{i}$ is the "true" value of the observable. Equation (9) defines nuisance parameters, $\delta_{i}$ and $\lambda_{\alpha}$. These will be random numbers with mean value 0 and standard deviation 1 ,

$$
\begin{aligned}
& \left\langle\delta_{i}\right\rangle=0 \quad \text { and } \quad\left\langle\delta_{i}^{2}\right\rangle=1 \\
& \left\langle\lambda_{\alpha}\right\rangle=0 \quad \text { and } \quad\left\langle\lambda_{\alpha}^{2}\right\rangle=1 .
\end{aligned}
$$

We assume that the uncorrelated errors are completely uncorrelated among the $N$ measurements; that is,

$$
\left\langle\delta_{i} \delta_{j}\right\rangle=\delta_{i j}
$$

We also assume that the systematic errors are completely correlated among the $N_{p t}$ measurements; that is, $\lambda_{\alpha}$ does not depend on $i$. However, the systematic errors are among themselves uncorrelated,

$$
\left\langle\lambda_{\alpha} \lambda_{\beta}\right\rangle=\delta_{\alpha \beta}
$$

The goal of the global analysis is to find the theoretical parameters for which the theoretical values $T_{i}$ of the observables are as close as possible to the "true" values, $X_{i}$. In Eq. (9) replace $X_{i}$ by $T_{i}$. Then we are led to minimize the differences, by defining

$$
\chi_{E}^{2}(\{a\},\{\lambda\})=\chi_{D}^{2}+\chi_{\lambda}^{2},
$$

where

$$
\chi_{D}^{2} \equiv \sum_{k=1}^{N_{p t}} \frac{1}{s_{k}^{2}}\left(D_{k}-T_{k}-\sum_{\alpha=1}^{N_{\lambda}} \beta_{k, \alpha} \lambda_{\alpha}\right)^{2}
$$

and

\footnotetext{
${ }^{7}$ The "true" value is the mean value of $D_{i}$ that would result from a large number of independent experiments.
}

$$
\chi_{\lambda}^{2} \equiv \sum_{\alpha=1}^{N_{\lambda}} \lambda_{\alpha}^{2}
$$

and minimizing $\chi_{E}^{2}$ with respect to the theory parameters. However, there are additional sources of systematic uncertainties associated with the unknown nuisance parameters $\left\{\lambda_{\alpha}\right\}$. Therefore, to have agreement between theory and data within the standard deviations of the experimental errors, we also vary the $\left\{\lambda_{\alpha}\right\}$ values, seeking to make $\chi_{E}^{2}$ small.

Because $\chi_{E}^{2}$ is only a quadratic function of $\left\{\lambda_{\alpha}\right\}$, we may obtain the minimum of $\chi_{E}^{2}$ with respect to $\left\{\lambda_{\alpha}\right\}$ analytically [65]. At the minimum, the nuisance parameters take the values

$$
\bar{\lambda}_{\alpha}=\sum_{i=1}^{N_{p t}} \frac{D_{i}-T_{i}}{s_{i}} \sum_{\delta=1}^{N_{\lambda}} \mathcal{A}_{\alpha \delta}^{-1} \frac{\beta_{i, \delta}}{s_{i}},
$$

where

$$
\mathcal{A}_{\alpha \beta}=\delta_{\alpha \beta}+\sum_{k=1}^{N_{p t}} \frac{\beta_{k, \alpha} \beta_{k, \beta}}{s_{k}^{2}} .
$$

The corresponding best-fit representation for $\chi_{E}^{2}$ is

$$
\min \chi_{E}^{2}=\sum_{i, j}^{N_{p t}}\left(D_{i}-T_{i}\right)\left(\operatorname{cov}^{-1}\right)_{i j}\left(D_{j}-T_{j}\right) .
$$

It includes the inverse of the covariance matrix

$$
(\mathrm{cov})_{i j} \equiv s_{i}^{2} \delta_{i j}+\sum_{\alpha=1}^{N_{\lambda}} \beta_{i, \alpha} \beta_{j, \alpha},
$$

given by

$$
\left(\operatorname{cov}^{-1}\right)_{i j}=\left[\frac{\delta_{i j}}{s_{i}^{2}}-\sum_{\alpha, \beta=1}^{N_{\lambda}} \frac{\beta_{i, \alpha}}{s_{i}^{2}} \mathcal{A}_{\alpha \beta}^{-1} \frac{\beta_{j, \beta}}{s_{j}^{2}}\right] .
$$

The best-fit value of $\chi_{D}^{2}$ can also be expressed in terms of the data $D_{s h, i}$ that are "shifted" from the central values by the best-fit correlated errors,

$$
\min \chi_{D}^{2}=\sum_{i=1}^{N_{p t}}\left(D_{s h, i}-T_{i}\right)^{2} / s_{i}^{2}
$$

Here

$$
D_{s h, i}=D_{i}-\sum_{\alpha=1}^{N_{\lambda}} \beta_{i \alpha} \bar{\lambda}_{\alpha} .
$$

We quote the function $\min \chi_{D}^{2}$ in Table I as a useful measure of the agreement between theory and data for each 
experiment. In Sec. VI, we will also use $\chi_{E}^{2}=\chi_{D}^{2}+\chi_{\lambda}^{2}$ as another measure of the agreement between theory and data.

Thus far, we have considered only a single experiment. The global chi-square function that is minimized in the CT10 global analysis sums over all experiments $E$ and includes a contribution $\chi_{t h}^{2}(\{a\})$ specifying theoretical conditions for the PDF parameters,

$$
\chi_{\text {global }}^{2}=\sum_{\mathrm{E}} \chi_{E}^{2}+\chi_{\text {th }}^{2} .
$$

The function $\chi_{\text {th }}^{2}$ is introduced to prevent some unconstrained PDF parameters from reaching values that might lead to unphysical predictions at small Bjorken $x$ (specifically, $x \leq 10^{-4}$ ), where experimental constraints are sparse [4]. It rules out those PDF parameter combinations that may result in negative cross sections or unlikely flavor dependence. The specific condition imposed in the CT10 analysis is to constrain the ratio $R_{s}(x, Q)=$ $[s(x, Q)+\bar{s}(x, Q)] /[\bar{u}(x, Q)+\bar{d}(x, Q)]$ of the strange PDFs to nonstrange sea PDFs to be in the interval $0.5 \leq$ $R_{s}\left(x, Q_{0}\right) \leq 1.5$ at the initial scale $Q_{0}$ and $x$ below $10^{-5}$. In the current fit, the $R_{s}$ ratio is not constrained in this region by the experimental data; hence a loose theory-motivated constraint needs to be imposed.

The global $\chi^{2}$ function in Eq. (24) is constructed by assuming that both uncorrelated and correlated errors are quasi-Gaussian and symmetric. When an experiment provides asymmetric errors, we symmetrize them. The exact procedure for symmetrization of errors has a low impact on the outcome of the fit, as the number of points with very asymmetric errors typically is small compared to the total number of points and parameters.

\section{B. PDF eigenvector sets and $\alpha_{s}$ uncertainty}

Besides the central (best-fit) PDF set, the CT10NNLO release includes PDF eigenvector sets to estimate the uncertainty range of our PDF fits using the Hessian method $[65,88]$ and either the symmetric [1] or the asymmetric $[10,89]$ master formula to estimate the PDF uncertainties. The Hessian method is based on an iterative procedure for finding linear combinations of the fitting parameters by diagonalization of the Hessian matrix. In the CT10NNLO fit, we use 25 free parameters to describe the parton distributions at $Q_{0}$, and hence have 25 eigenvector directions. For each of these directions, we find a pair of eigenvector sets defined by moving away from the best-fit location (where $\chi^{2}$ takes its minimum value) by a distance that estimates the boundary of the $90 \%$ confidence interval for each experiment.

The method to find these eigenvectors is explained in [10]. In addition to including an upper "tolerance" bound on the increase in total $\chi^{2}$ that realizes the $90 \%$ C.L. agreement on average, as in the CTEQ6 analysis, we include a penalty term in $\chi^{2}$ that quickly grows when the PDF set fails to describe any specific experiment. The effective function $\chi_{\mathrm{Eff}}^{2}$ that is constructed this way is scanned along each eigenvector direction until $\chi_{\text {Eff }}^{2}$ increases above the tolerance bound or quick $\chi_{\text {Eff }}^{2}$ growth due to the penalty is triggered.

The penalty term is constructed from statistical variables $S$ derived from $\chi_{E}^{2}$ values for individual experiments [10]. In contrast to $\chi_{E}^{2}$, the variables $S$ obey an approximate standard normal distribution independently of $N_{p t}$, which simplifies the comparison of confidence levels between data sets containing widely different numbers of points. The quasi-Gaussian $S$ variables are found by using a simpler Fisher's approximation [90] in the CT10 NLO fit (reliable for experiments with many data points, $\left.N_{p t} \gtrsim 9\right)$ and more accurate Lewis's approximation [91] in the CT10NNLO analysis.

We note that even in the central fit, some data sets have $\chi_{E}^{2} / \mathrm{N}_{p t}$ that lie outside the $90 \%$ confidence level. That is not surprising, of course, since there are 28 data setsnaively the chance for all of them to lie with $90 \%$ confidence is only $0.9^{28}=0.05$. To allow for this, for each data set that has $\chi_{E}^{2}>N_{p t}$ in the central fit, we rescale its $\chi_{E}^{2}$ by a factor $N_{p t} / \chi_{\text {centralfit }}^{2}$ before computing the penalty.

In practical applications, the PDF uncertainty obtained with the Hessian eigenvector sets must be combined with the $\alpha_{s}$ uncertainty. The procedure for combining these uncertainties is described in [36]. The central and error eigenvector sets of the CT10NNLO family assume $\alpha_{s}\left(M_{Z}\right)=0.118$, which is compatible with the worldaverage value. In addition we provide best-fit PDF sets for other $\alpha_{s}\left(M_{Z}\right)$ values in the interval 0.112-0.127. For a theory observable, the $90 \%$ C.L. $\alpha_{s}$ uncertainty can be estimated as the half of the difference of predictions using PDF sets with $\alpha_{s}=0.116$ and 0.120 . Then, the PDF $+\alpha_{s}$ uncertainty with all correlations can be estimated by adding the PDF and $\alpha_{s}$ uncertainties in quadrature.

\section{Implementation of correlated systematic errors}

In the treatment of correlated systematic errors, there is another subtlety concerned with the distinction between additive and multiplicative systematic uncertainties. The correlated systematic errors fall into two classes: additive errors, for which the experiment can determine the absolute value $\beta_{i, \alpha}$ of the standard deviation; or multiplicative errors, for which only the relative fraction $\sigma_{i, \alpha}=\beta_{i, \alpha} / X_{i}$ is known. The two kinds must be handled differently to avoid a bias in the outcome of the fit. However, it is a common practice in many experiments to publish the correlated systematic uncertainties as the relative percentage errors $\sigma_{i, \alpha}$ regardless of whether the systematic error is additive or multiplicative, rather than the absolute values $\beta_{i, \alpha}$.

To reconstruct the correlation matrix as

$$
\beta_{i, \alpha}=\sigma_{i, \alpha} X_{i}
$$


one selects the reference central value $X_{i}$ for each datum. Since the additive errors are supposedly independent of the theory predictions, it is natural, although not necessary, to use the experimental central values $D_{i}$ as the references, $\beta_{i, \alpha}=\sigma_{i, \alpha} D_{i}$.

For a multiplicative error, the reference to $D_{i}$ is generally unacceptable, as random fluctuations in $D_{i}$ tend to bias the best-fit parameters. A well-known multiplicative bias described by D'Agostini arises in the treatment of the normalization of the data $[92,93]$. A fit that references the normalization error to $D_{i}$ would underestimate the true cross section. This downward bias is prevented if smoothly behaving $X_{i}$ values are used as the references, such as the theoretical values $T_{i}$ at each data point $\left(\beta_{i, \alpha}=\sigma_{i, \alpha} T_{i}\right)$.

Most of the time the experimental paper does not distinguish between the additive and multiplicative errors. In this case, the global fit has to choose between several ways, and several trade-offs, for computing $\beta_{i, \alpha}$. When preparing the CT10 NNLO PDFs, we explored various procedures for the computation of $\beta_{i, \alpha}$ that have been identified in recent literature. (See also a related discussion in the appendix of Ref. [94].)

1. Method $D$, or the "experimental normalization" method, normalizes all correlated errors to the experimental central values: that is, we compute $\beta_{i, \alpha}=\sigma_{i, \alpha} D_{i}$, for all additive and multiplicative errors alike. In this method, the correlated errors are independent of theory predictions. However, they are affected by irregular fluctuations of the central data points, which may result in a pronounced D'Agostini bias in the best-fit parameters when the fluctuations are large.

2. Method $T$ normalizes the multiplicative errors to the theoretical values that are updated in every fitting iteration. "The $T^{(0)}$ method" [95] is a variation on this approach in which the multiplicative errors are updated once in many iterations. The $T^{(0)}$ method was proposed to prevent nonlinear behavior of $\chi^{2}$ that may occur in method $T$. Additive errors in these methods remain normalized to $D_{i}$. Both methods $T$ and $T^{(0)}$ are free of D'Agostini's bias, as the multiplicative errors are estimated by using a smooth function. Method $T^{(0)}$ converges to the true solution after several updates of the $T_{i}^{(0)}$ values, provided the partial derivatives $\partial \chi^{2} / \partial T_{i}^{(0)}$ are negligible, and may fail to do so otherwise. Our comparisons follow the original implementation of the $T$ and $T^{(0)}$ methods [95], in which only the luminosity errors (but not other multiplicative errors such as for the jet energy scale) were referenced to $T_{i}\left(T_{i}^{(0)}\right)$.

3. An extended version of method $T$ normalizes both additive and multiplicative errors to the current theoretical values, $\beta_{i, \alpha}=\sigma_{i, \alpha} T_{i}$. Similarly, the extended method $T^{(0)}$ normalizes all correlated errors to fixed theoretical values, $\beta_{i, \alpha}=\sigma_{i, \alpha} T_{i}^{(0)}$. The advantage of these methods is that $\beta_{i, \alpha}$ shows the smoothest behavior among all considered.

The extended method $T$ is used by default in our NLO fits, including all CTEQ6.X series and CT10(W) NLO. At NNLO the implementation of systematic effects became even more important, given the reduction in other uncertainties. In Sec. VID we compare five procedures for implementation of systematic errors in inclusive jet production, where the systematic effects are among the most pronounced. We use the extended $T$ method for other scattering processes.

\section{EXPERIMENTAL DATA SETS AND THEORETICAL UPDATES}

\section{A. Data selection: Vector boson and jet production}

The experimental data sets included in the CT10 NNLO fit are listed in Table I. With a small number of exceptions, they were chosen to be the same as in the CT10 NLO fit. At NLO, we presented two PDF sets, designated as CT10 and CT10W. The distinction between them concerns the inclusion of the D0 Run-2 data for the rapidity asymmetry $\left(A_{\ell}\right)$ of the charged lepton from $W$ boson decay. These data were included in the CT10W analysis, with an extra weight, but not included in the CT10 analysis. More specifically, the CT10W analysis includes the $A_{\ell}$ data points in three ranges of the lepton transverse momentum $\left(p_{T \ell}\right)$ in the electron decay channel and one $p_{T \ell}$ bin in the muon decay channel. After the publication of the CT10 and CT10W analyses, the D0 Collaboration has recommended not to include those two less inclusive data sets in $p_{T \ell}$, i.e., with $25 \leq p_{T \ell} \leq$ $35 \mathrm{GeV}$ and $p_{T \ell} \geq 35 \mathrm{GeV}$. In the CT10NNLO analysis, only the most inclusive data with $p_{T \ell} \geq 25 \mathrm{GeV}$, in both the electron and muon decay channels, are included in the analysis. Since CT10NNLO includes only a part of the D0 $A_{\ell}$ data that distinguishes between CT10 and CT10W PDFs, it can be treated as an NNLO counterpart to either the CT10 or CT10W NLO PDF sets.

The Tevatron Run-2 inclusive jet data have a wider rapidity coverage (in the case of $\mathrm{CDF}$ ) and smaller statistical and systematic errors, as compared to the Tevatron Run-1 data. Their effects on the global analysis have been extensively discussed in Ref. [5]. We concluded that since the Run-1 jet data are not perfectly consistent with the Run-2 data sets, one may ask if the Run-1 jet contributions should be retained. In the CT10NNLO analysis, we do not include the Tevatron Run-1 inclusive jet data. This choice has implications for the large- $x$ gluon PDF, as will be shown later.

The data selection choices in regard to the Tevatron $W$ asymmetry and inclusive jet production affect mainly certain combinations of the PDFs at $x>0.1$ : specifically the ratio $d / u$ (down- versus up-quark PDFs) and the gluon PDF. These combinations change by amounts that are 
TABLE I. Experimental data sets examined in the CT10NNLO and CT10W NLO analyses, together with their $\chi^{2}$ values.

\begin{tabular}{|c|c|c|c|}
\hline Experimental data set & $N_{p t}$ & CT10NNLO & CT10W \\
\hline Combined HERA1 NC and CC DIS [66] & 579 & 1.07 & 1.17 \\
\hline BCDMS $F_{2}^{p}[67]$ & 339 & 1.16 & 1.14 \\
\hline BCDMS $F_{2}^{d}[68]$ & 251 & 1.16 & 1.12 \\
\hline NMC $F_{2}^{p}[69]$ & 201 & 1.66 & 1.71 \\
\hline NMC $F_{2}^{d} / F_{2}^{p}[69]$ & 123 & 1.23 & 1.28 \\
\hline CDHSW $F_{2}^{p^{2}}[70]$ & 85 & 0.83 & 0.66 \\
\hline CDHSW $F_{3}^{p}[70]$ & 96 & 0.81 & 0.75 \\
\hline $\mathrm{CCFR} F_{2}^{p}[71]$ & 69 & 0.98 & 1.02 \\
\hline CCFR $x F_{3}^{p}[72]$ & 86 & 0.40 & 0.59 \\
\hline NuTeV neutrino dimuon SIDIS [73] & 38 & 0.78 & 0.94 \\
\hline NuTeV antineutrino dimuon SIDIS [73] & 33 & 0.86 & 0.91 \\
\hline CCFR neutrino dimuon SIDIS [74] & 40 & 1.20 & 1.25 \\
\hline CCFR antineutrino dimuon SIDIS [74] & 38 & 0.70 & 0.78 \\
\hline $\mathrm{H} 1 F_{2}^{c}[75]$ & 8 & 1.17 & 1.26 \\
\hline $\mathrm{H} 1 \sigma_{r}^{c}$ for $c \bar{c}[59,76]$ & 10 & 1.63 & 1.54 \\
\hline ZEUS $F_{2}^{c}[57]$ & 18 & 0.74 & 0.90 \\
\hline ZEUS $F_{2}^{c}[58]$ & 27 & 0.62 & 0.76 \\
\hline E605 Drell-Yan process, $\sigma(p A)[77]$ & 119 & 0.80 & 0.81 \\
\hline E866 Drell Yan process, $\sigma(p d) /(2 \sigma(p p))[78]$ & 15 & 0.65 & 0.64 \\
\hline E866\NuSea Drell-Yan process, $\sigma(p p)[79]$ & 184 & 1.27 & 1.21 \\
\hline CDF Run-1 W charge asymmetry [80] & 11 & 1.22 & 1.24 \\
\hline CDF Run- $2 W$ charge asymmetry [81] & 11 & 1.04 & 1.02 \\
\hline D $\varnothing$ Run-2 $W \rightarrow e \nu_{e}$ charge asymmetry [82] & 12 & 2.17 & 2.11 \\
\hline DØ Run-2 $W . \mu \nu_{\mu}$ charge asymmetry [83] & 9 & 1.65 & 1.49 \\
\hline DØ Run-2 Z rapidity distribution [84] & 28 & 0.56 & 0.54 \\
\hline CDF Run-2 Z rapidity distribution [85] & 29 & 1.60 & 1.44 \\
\hline CDF Run-2 inclusive jet production [86] & 72 & 1.42 & 1.55 \\
\hline DØ Run-2 inclusive jet production [87] & 110 & 1.04 & 1.13 \\
\hline Total: & 2641 & 1.11 & 1.13 \\
\hline
\end{tabular}

mostly comparable to the PDF uncertainties obtained in the NLO analysis.

\section{B. Data selection: Fixed-target DIS experiments}

As in CT10(W) NLO, the inclusive proton data from BCDMS, NMC, CDHSW, and CCFR experiments are included in the form of structure functions $F_{2}(x, Q)$. Those were derived by the experimental groups from the measured cross sections using the ratio $R=\sigma_{L} / \sigma_{T}$ of the longitudinal to transverse cross sections for virtual photon DIS that is known better now than at the time of the experimental publications. In the past candidate fits, we have reconstructed $F_{2}(x, Q)$ using an alternative $R$ parametrization or replaced it entirely by a reduced DIS cross section when available. The resulting modifications in the PDFs in these trials have not exceeded the published PDF uncertainty.

In the CT10 NNLO analysis, the replacement of the BCDMS $F_{2}(x, Q)$ by the respective reduced cross sections ${ }^{8}$

\footnotetext{
${ }^{8}$ The reduced cross sections are reconstructed from the BCDMS data on $F_{2}^{p, d}(x, Q)$ determined with the assumption of $R=0$
}

(without refitting) results in about the same fit quality: $\chi^{2} / N_{p t}=1.12$ and 1.24 for the BCDMS proton and deuteron reduced cross sections, to be compared against 1.16 and 1.16 for the data on $F_{2}^{p, d}(x, Q)$.

Recently, the ABM group found that the replacement of the NMC reduced cross section for inclusive DIS on the proton by the corresponding $F_{2}^{p}(x, Q)$ data modifies the preferred gluon distribution and $\alpha_{s}\left(M_{Z}\right)$ in their NNLO fit, as well as the Higgs cross section that depends on them [96]. We performed a similar comparison in the context of the CT10 NNLO study, by replacing $F_{2}^{p}(x, Q)$ for NMC (our default choice) by the NMC reduced cross section. It was known for a long time that the NMC $F_{2}^{p}$ data are not fitted well in CTEQ analyses [1], for reasons that are not completely understood. The CT10 NNLO fit results in $\chi^{2} / N_{p t}=1.67$ for the $\mathrm{NMC}_{2}^{p}(x, Q)$ data. In comparison, the $\chi^{2} / N_{p t}$ value for the NMC reduced cross section data is

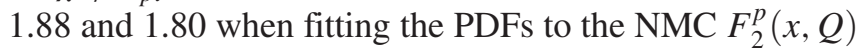
and NMC reduced cross section, respectively. It is not possible to get a good fit to the NMC proton data set even if its statistical weight in the $\chi^{2}$ is increased or other fixed-target DIS experiments (BCDMS) are dropped from the fit. 
For $F_{2}^{p}(x, Q)$ data, the NNLO fit to the NMC data behaves similarly to the CTEQ6 NLO fit discussed in Appendix B.2 of [1]. The distribution of data-theory residuals is consistent with larger-than-normal fluctuations of the data. All 12 nuisance parameters for experimental systematic shifts are below 1.5 and contribute $\chi_{\lambda}^{2} \approx 6$ to $\chi^{2}$. In contrast, a fit to the NMC reduced cross section results in theory typically overshooting the data and requires $6 \sigma$ and $2.8 \sigma$ shifts in the experimental radiative correction and luminosity for the NMC set at the global minimum of $\chi^{2}$.

For the world-average $\alpha_{s}\left(M_{Z}\right)=0.118$ adopted by CT10 NNLO, the above replacement induces essentially no change in the best-fit PDFs. When $\alpha_{s}\left(M_{Z}\right)$ is varied, the NMC reduced cross section is still poorly fitted and prefers $\alpha_{s}\left(M_{Z}\right)=0.112-0.113$, i.e., much lower than the worldaverage value $\alpha_{s}\left(M_{Z}\right)=0.1184 \pm 0.0007$ [64]. We therefore choose to fit the NNLO PDFs to the NMC $F_{2}^{p}(x, Q)$ data for consistency with CT10 NLO, given that the replacement of $F_{2}^{p}(x, Q)$ by the reduced cross section neither improves the fit nor modifies the PDFs. Besides the above-mentioned observations about the NMC data made in the CTEQ6 NLO study, the MSTW [97] and NNPDF [98] groups have reached a similar conclusion that some NMC data sets are poorly fitted, and the replacement of the structure functions by the reduced cross sections for them is inconsequential for the NNLO PDFs.

\section{Theory developments}

Besides implementing the NNLO QCD contributions in neutral-current DIS and vector boson production, we updated the theoretical treatment of several experiments. In the coefficient functions for deep inelastic scattering, we have updated the definitions of the electroweak couplings describing $Z$ and $\gamma^{*}-Z$ interference contributions. This update mildly modifies the $d$ quark PDFs at large $x$.

In the previous global analyses, the NLO jet cross sections utilized tables of point-by-point ratios of NLO/ LO cross sections ( $K$ factors) computed with the EKS code [99]. The original EKS calculation was published in the 1990s and had limited accuracy when comparing it to the latest precision jet data. A deeply modified version of the EKS code (MEKS) was prepared to provide advanced predictions for jet cross sections [100]. The MEKS calculation is entirely independent from NLOJET++ [101], another frequently used program for computation of NLO jet cross sections. A comparison to MEKS validated predictions from FASTNLO [102-104] and APPLGRID [105], the interfaces for fast interpolation of NLOJET++ cross sections. The specific input settings for which the MEKS, FASTNLO, and APPLGRID programs agree to a few percent were documented $[94,100]$. The magnitude of theoretical uncertainties in inclusive jet cross sections was also estimated. It was observed, for example, that the current NLO QCD scale dependence is too large at the highest $P_{T j}$ and $y_{j}$ of the LHC inclusive jet data set to provide meaningful constraints on the relevant PDFs. This situation is expected to be improved when the NNLO inclusive jet cross section calculation becomes available.

In the CT10NNLO fit, we compute the NLO inclusive jet cross sections with FASTNLO version 1.0 and cross validate the results using MEKS. Some NNLO fits, such as MSTW and NNPDF, approximate the two-loop contribution to Tevatron inclusive jet production by expanding the correspondent logarithmic terms in the threshold resummation calculation [106]. Our jet cross sections are evaluated at NLO and do not include the threshold resummation contribution, as collider jet production is not dominated by the threshold kinematic region, and there are known examples when the exact NNLO correction is very different from its threshold approximation because of the substantial powerlike finite contributions arising in the exact NNLO result (see, e.g., $[107,108])$. Furthermore, at the LHC, the NLO cross section combined with a two-loop threshold contribution provided by FastNLO disagrees with the inclusive jet data [94].

In the jet cross sections, both the factorization and renormalization scales are chosen to be equal to the transverse momentum $P_{T j}$ of each individual jet. This choice is different from the one adopted in the CTEQ6.X and CT10 NLO analyses, where both scales were set to $P_{T j} / 2$.

\section{THE CT10NNLO PARTON DISTRIBUTION FUNCTIONS}

With a similar setup as in the CT10/CT10W NLO analysis, the CT10NNLO fit results in about the same quality of the fit expressed in terms of the total chi square. We obtain $\chi^{2}$ of order 2950 for 2641 data points, with minor variations dependent on the setup of the fit. In the rest of the paper, we discuss representative results from the new fit, assuming NNLO PDFs, unless stated otherwise.

The central fit and PDF eigenvector sets are shown in Fig. 2. In addition to obtaining the central fit, there is also a need to understand the uncertainty ranges of the PDFs, resulting from the experimental uncertainties of the data included in the fit. For this purpose, we generate 50 alternate fits using the Hessian method. Figure 2 indicates the PDFs for the alternate fits, also called the "error PDFs," in addition to the central fit. In each graph, PDFs for four parton combinations are shown, by plotting $x f(x, Q)$ versus $x$ for a fixed value of $Q$. The parton combinations are $f=u_{\text {valence }}, d_{\text {valence }}, g, q_{\text {sea }}$. The values of $Q$ are 2, 3.16, $8,85 \mathrm{GeV}$. The dashed curves are the corresponding functions for the CT10 NLO PDFs. Those can differ by significant amounts from the NNLO ones in some regions of the $(x, Q)$ space, especially where $x$ and $Q$ are small.

To better assess these differences, Fig. 3 shows the ratios of various parton distributions from the CT10NNLO central fit to those from the CT10W NLO central fit, at $Q=2 \mathrm{GeV}$. A few changes are noticeable: (1) At 
$\mathbf{x} f(x, Q)$ versus $x$
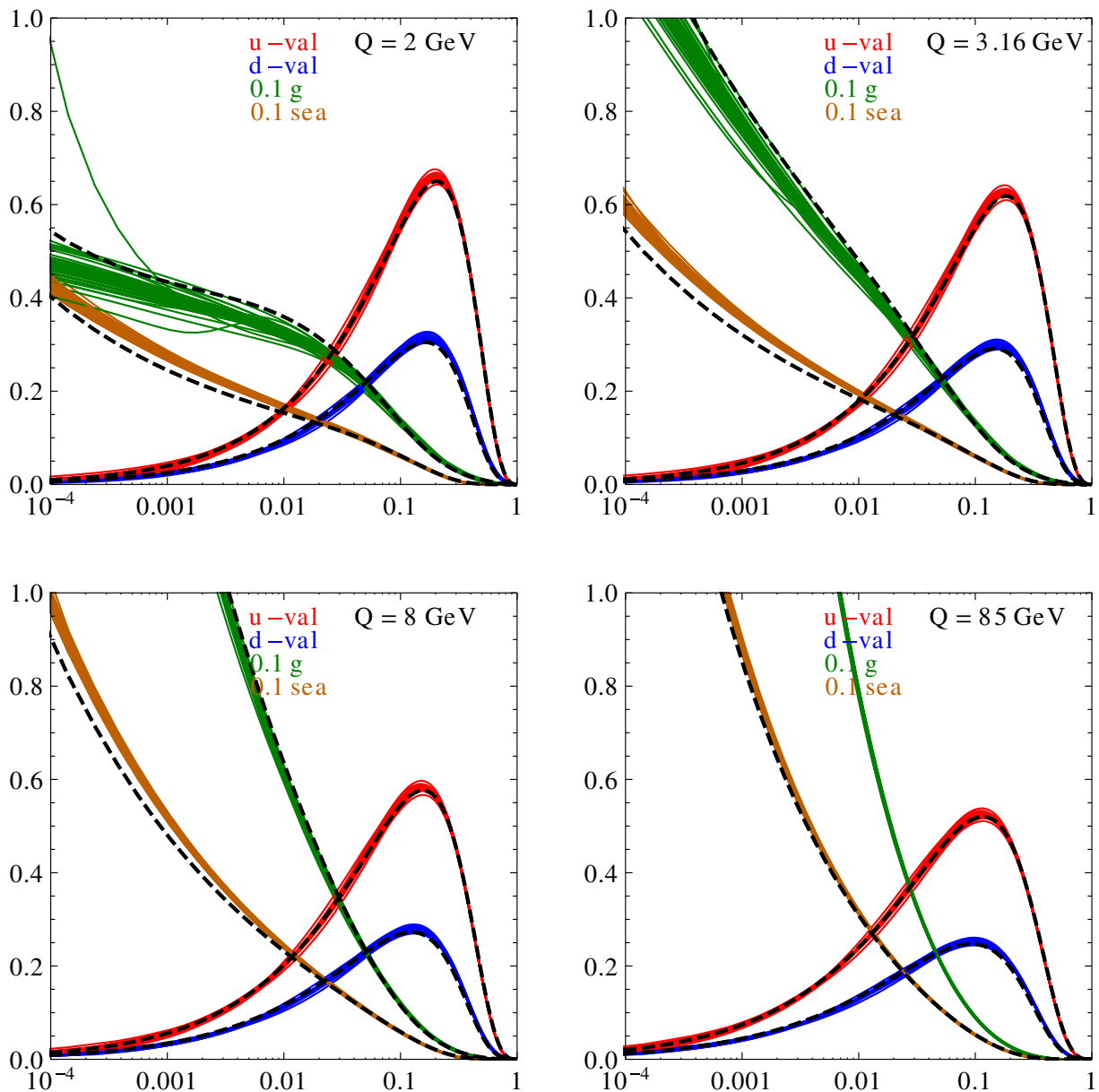

FIG. 2 (color online). CT10NNLO parton distribution functions. These figures show the Hessian error PDFs from the CT10NNLO analysis. Each graph shows $x u_{\text {valence }}=x(u-\bar{u}), x d_{\text {valence }}=x(d-\bar{d}), 0.10 x g$ and $0.10 x q_{\text {sea }}$ as functions of $x$ for a fixed value of $Q$. The values of $Q$ are $2,3.16,8,85 \mathrm{GeV}$. The quark sea contribution is $q_{\text {sea }}=2(\bar{d}+\bar{u}+\bar{s})$. The dashed curves are the central CT10 NLO fit.

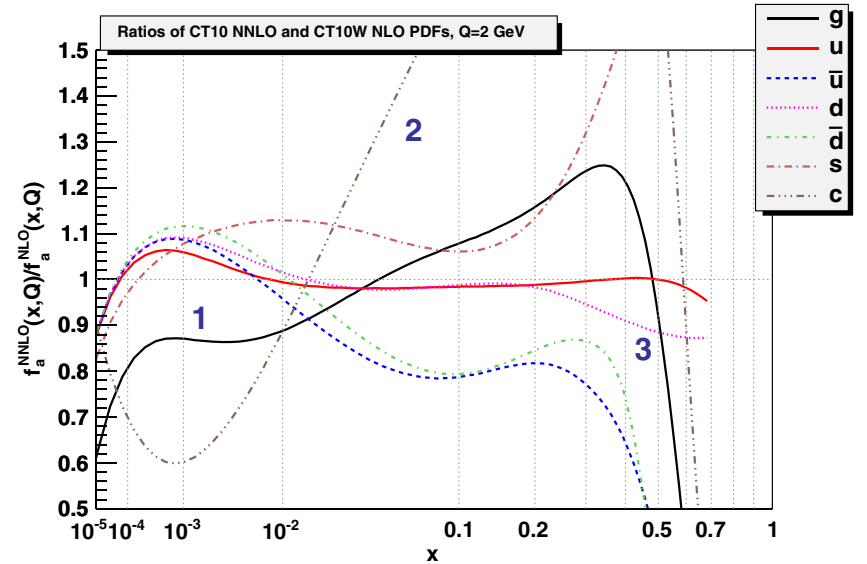

FIG. 3 (color online). Ratios of various CT10NNLO central fit parton distributions to those of the CT10W central fit, at $Q=2 \mathrm{GeV}$.

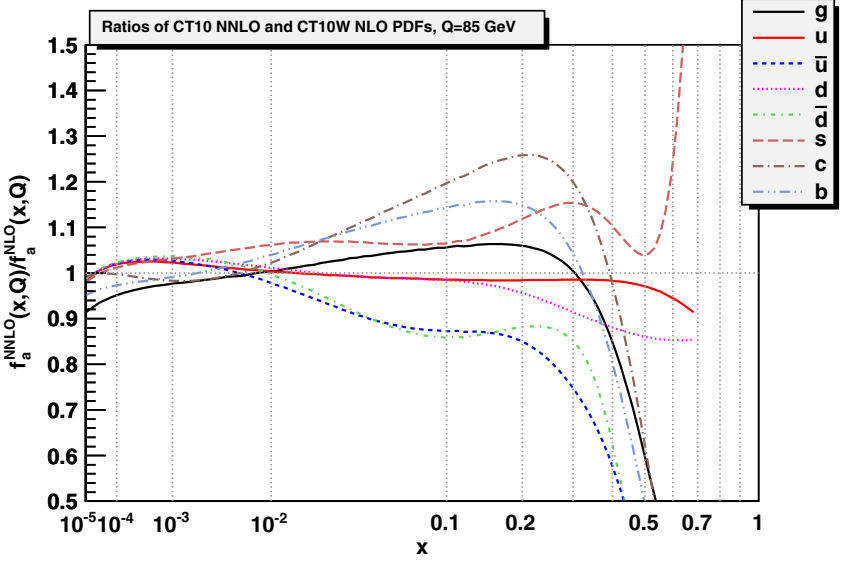

FIG. 4 (color online). Same as Fig. 3, at $Q=85 \mathrm{GeV}$. 

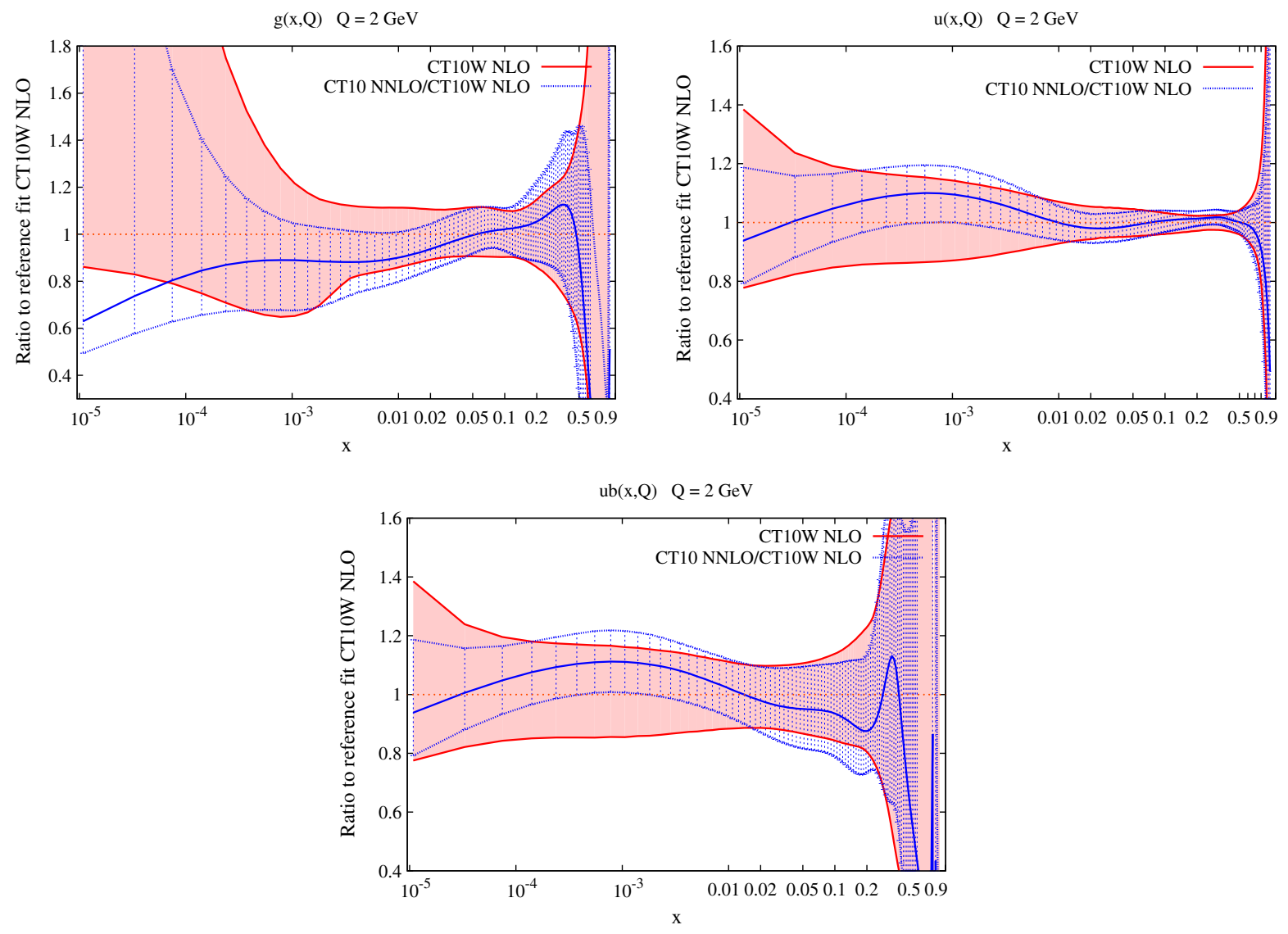

FIG. 5 (color online). Comparison of CT10NNLO error PDFs to CT10W (NLO) error PDFs. All are expressed as ratios to the central fit of the CT10W set.

$x<10^{-2}$, the $\mathcal{O}\left(\alpha_{s}^{2}\right)$ evolution in CT10NNLO suppresses $g(x, Q)$ and increases $q(x, Q)$, as compared to CT10W PDFs. (2) The heavy charm $c(x, Q)$ and bottom $b(x, Q)$ partons change as a result of adopting the $\mathcal{O}\left(\alpha_{s}^{2}\right)$ GM-VFN scheme in the CT10NNLO analysis. (3) In the large- $x$ region, $g(x, Q)$ and $d(x, Q)$ are reduced by the removal of

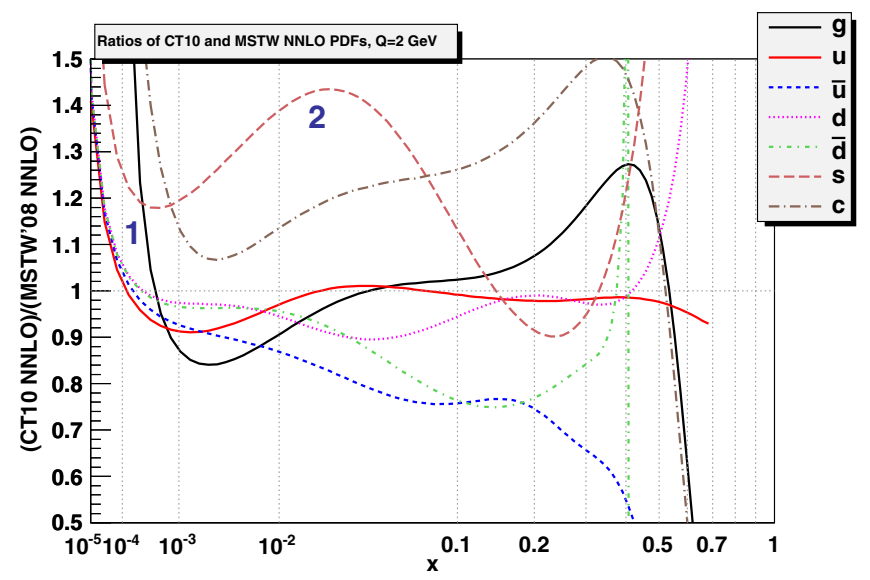

FIG. 6 (color online). Ratios of various CT10NNLO central fit parton distributions to those of the MSTW2008NNLO central fit, at $Q=2 \mathrm{GeV}$. the Tevatron Run-1 inclusive jet data, the revised electroweak couplings, the alternative treatment of correlated systematic errors, and the scale choices.

A similar comparison, but for $Q=85 \mathrm{GeV}$, is shown in Fig. 4. It also indicates that at $x>0.1, d(x, Q), \bar{u}(x, Q)$ and $\bar{d}(x, Q)$ are all reduced in CT10NNLO, compared to CT10W. Likewise, $g(x, Q)$ is also reduced in the large $x$ region.

The uncertainties of CT10NNLO PDFs for various parton flavors are shown in Fig. 5, compared to the CT10W (NLO) uncertainties, at $Q=2 \mathrm{GeV}$.

The CT10NNLO central fit is compared to that of MSTW2008NNLO [24] in Fig. 6. It shows that the CT10NNLO gluon and quarks are larger as $x \rightarrow 0$. At the smallest $x$ values and at the initial scale $Q_{0}$, the CT10 NNLO gluon remains positive, although consistent with zero, as a result of the chosen form of parametrization. We also note that CT10NNLO strangeness is larger at $x \sim 10^{-3}$.

\section{COMPARISONS TO INDIVIDUAL EXPERIMENTS}

As was already observed, the goodness of the NNLO fit to most experiments is about the same as for CT10(W) 
NLO, and the changes in going to the NNLO analysis are comparable to the experimental uncertainties. The differences in $\chi_{E}^{2}$ rarely exceed the expected statistical fluctuations of order $\sqrt{2 N_{p t}}$. Some improvement is observed in the fit to the HERA-1 combined data on DIS, the Tevatron Run- 2 jet production data, and the CCFR and $\mathrm{NuTeV}$ dimuon SIDIS. In this section, we present examples of the description of the data by the CT10 theoretical predictions, focusing on select precise measurements in DIS, $W$ boson production, and jet production.

\section{A. Deep-inelastic scattering at HERA}

The HERA combined data set for $e^{ \pm} p$ neutral current (NC) and charged current (CC) deep-inelastic scattering [66] has 579 measurements of the reduced cross section $\sigma_{\mathrm{r}}(x, Q)$, after applying our restrictions $Q \geq 2 \mathrm{GeV}$ and $W \geq 3.5 \mathrm{GeV}$, where the invariant mass of the hadronic final state is $W=Q \sqrt{1 / x-1}$.

In Fig. 7 we show the $e^{+} p$ NC DIS reduced cross sections (366 data points) along with their uncorrelated error bars. Data are divided into two blocks: small $x$ is shown on the left, i.e., $0<x<0.002$, and large $x$ is shown on the right, i.e., $0.002 \leq x \leq 0.65$. Figure 7 also shows theory curves based on the CT10NNLO parton distribution functions, superimposed on the HERA combined data. Note that there are systematic differences between the central data and the theory, as there should be due to the systematic uncertainties of the data. Our detailed comparison of data and theory must indicate whether the systematic differences are consistent with the published errors [29].

Data divided by theory for $e^{+} p$ NC DIS is shown in Fig. 8, in which we have a clearer illustration of the differences. The horizontal lines are marked by the corresponding values of $x$. The black dots are the central experimental values, shown along with uncorrelated error bars. The red dots are the optimally shifted data values, determined by the Hessian analysis. We observe that the systematic shifts bring data and theory into better agreement, as expected.

A histogram of the residuals of the shifted data, defined by

$\operatorname{Residual}_{i}=\left(D_{i}-\sum_{\alpha=1}^{N_{\lambda}} \beta_{i \alpha} \bar{\lambda}_{\alpha}-T_{i}\right) / s_{i} \equiv\left(D_{s h, i}-T_{i}\right) / s_{i}$,

is shown in Fig. 9. The $i$ th residual is defined by the difference between the optimally shifted data and the theory, normalized to the uncorrelated error. The total number of systematic errors is $N_{\lambda}=114$. The curve shown in Fig. 9 is the standard normal distribution, with no adjustable parameters: it has a mean $=0$, standard deviation $=1$, and integral $=1$. The close similarity between the histogram of residuals and the Gaussian curve implies that the data and theory agree within the experimental errors, and that the experimental errors have been correctly estimated.

The best-fit nuisance parameters $\left\{\bar{\lambda}_{\alpha}\right\}$ for the combined HERA data are shown in the histogram of Fig. 10. The 113 sources of experimental systematic errors contribute $\chi_{\lambda}^{2}=$ 60 for this experiment, so that many correlated systematic uncertainties have no effect on the agreement with theory and lead to null shifts in their respective nuisance parameters. This is reflected in the histogram of $\left\{\bar{\lambda}_{\alpha}\right\}$, which is clearly narrower than the standard normal distribution.

\section{B. $W$ charge asymmetry and vector boson production}

The $W$ charge asymmetry measured at the Tevatron is primarily sensitive to the shape of the ratio $d\left(x, M_{W}\right) / u\left(x, M_{W}\right)$ at $x$ values above 0.1 . The NNLO fit produces essentially the same quality of the agreement with the included Tevatron $W$ asymmetry sets as the CT10W NLO PDF set. The $\chi^{2} / N_{p t}$ values for these data sets are listed in Table I. As an illustration, Fig. 11 shows the CT10W NLO and CT10 NNLO data residuals for the 12 points of the D0 Run-2 lepton asymmetry data in the electron [82] and muon [83] decay channels. The residuals are very close for the two PDF sets. The quality of the NLO and NNLO fits to other data sets on vector boson production is also comparable.

\section{Inclusive jet production from Run-2 at the Tevatron}

The CT10NNLO global analysis only includes the Tevatron Run-2 inclusive jet data for the reasons explained in Sec. IV. In what follows we examine the goodness of CT10NNLO fits to the Run-2 inclusive jet data.

In Fig. 12 we show the differential cross section $d^{2} \sigma /\left(d y_{j} d P_{T j}\right)$ for inclusive jet production at D0 Tevatron Run-2 [87]. The various curves correspond to the six rapidity intervals $0-0.4,0.4-0.8,0.8-1.2,1.2-1.6$, 1.6-2.0, and 2.0-2.4. The points are the central data values, while the error bars (too small to be seen in most cases) represent uncorrelated errors. In the same figure, we also show the theory calculations based on the CT10NNLO parton distribution functions. In this figure we do not show systematic errors. With the systematic shifts, we obtain $\chi_{E}^{2} / N_{p t}=115 / 110$ for D0 Run-2, where 15 units are contributed by the penalty term $\chi_{\lambda}^{2}$ for the 23 nuisance parameters $\left(\lambda_{\alpha}\right)$ controlling the systematic shifts; cf. the definition in Eq. (14). The overall effect of the correlated systematic shifts is significant, compared to the small uncorrelated errors, and is of the same order as in the CT10 NLO fits. More detailed comparisons at NLO, illustrating the impact of the systematic shifts on the jet data, can be found in [5].

To check the consistency of the comparison of the shifted data with CT10NNLO predictions, Fig. 13 shows histograms of the residuals, as defined in Eq. (26), for six bins of the jet rapidity $(y)$. The distributions observed in the 


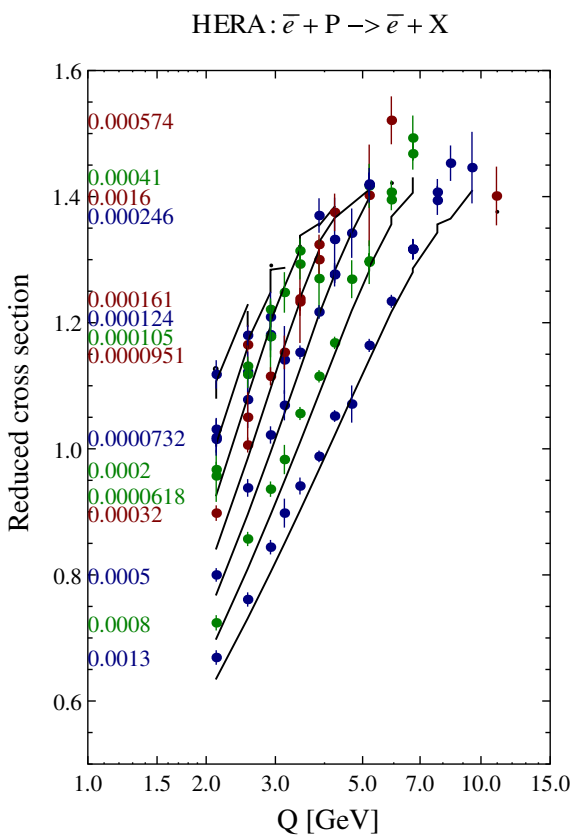

HERA $: \bar{e}+\mathrm{P}->\bar{e}+\mathrm{X}$

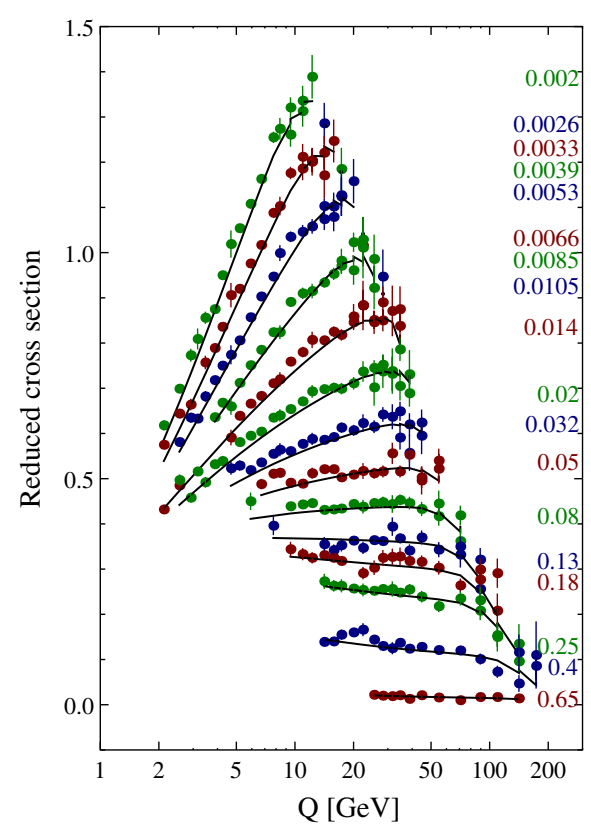

FIG. 7 (color online). The HERA combined data, with comparison to theory. The reduced cross section $\sigma_{r}(x, Q)$ for NC DIS of positrons is plotted as a function of $Q$ for 33 values of $x$. The graph on the left has $x$ values in the range $0<x<0.002$; the one on the right has $0.002 \leq x \leq 0.65$. The points are the published central values, while error bars correspond to the uncorrelated errors only. The red curves show the theoretical value of the reduced cross section $\sigma_{r}(x, Q)$ computed with CT10NNLO PDFs.
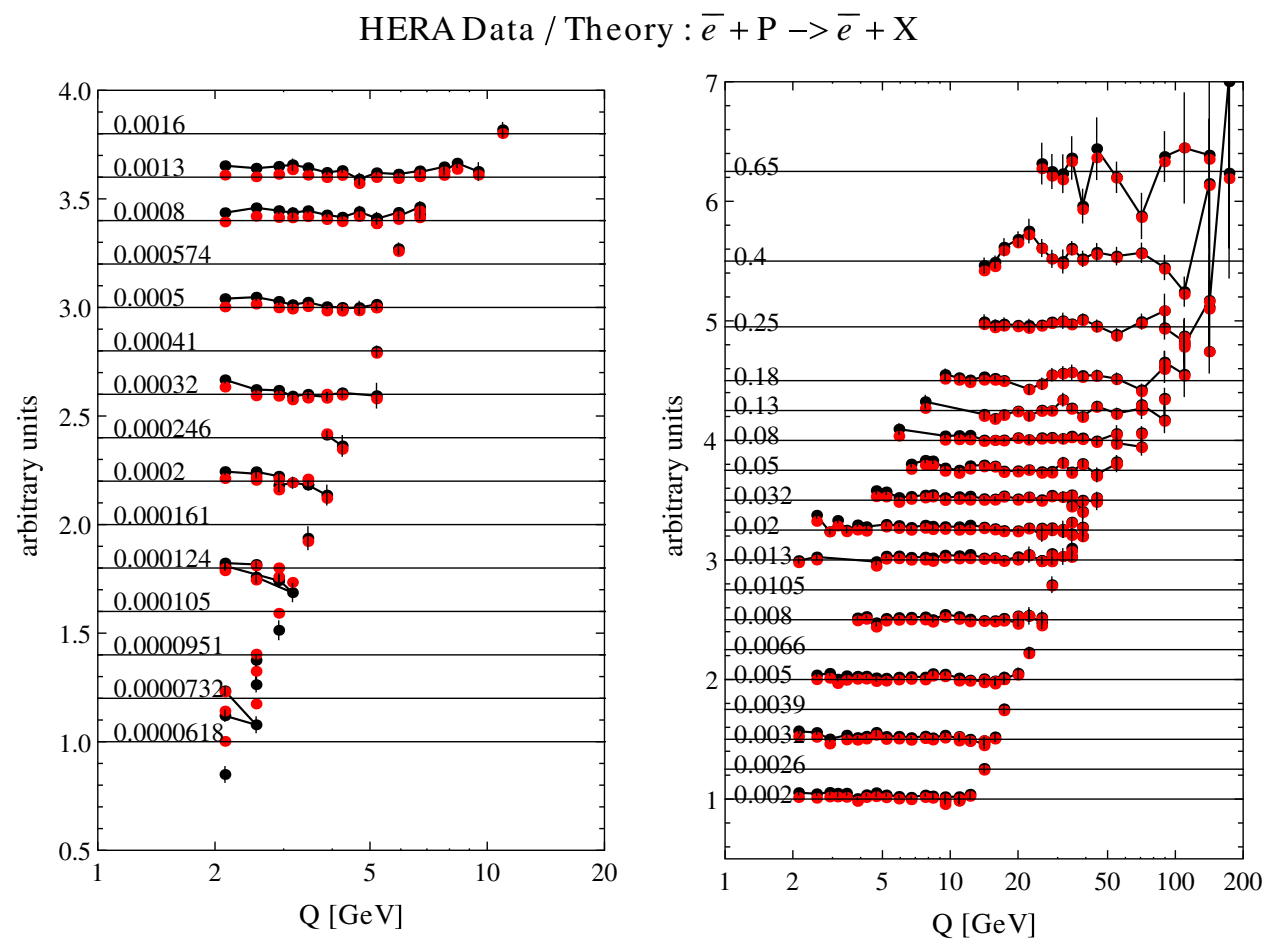

FIG. 8 (color online). Ratio of data divided by theory, for the reduced cross section for positron-proton NC DIS. Theory is represented by the horizontal lines. Indicated in both figures are the $x$ values: $0<x \leq 0.0016$ in the left inset; $0.002 \leq x \leq 0.65$ in the right inset. Black dots are experimental central values with uncorrelated errors only. Red dots are the optimally shifted data, where the shifts are evaluated according to the Hessian analysis of the systematic errors. 


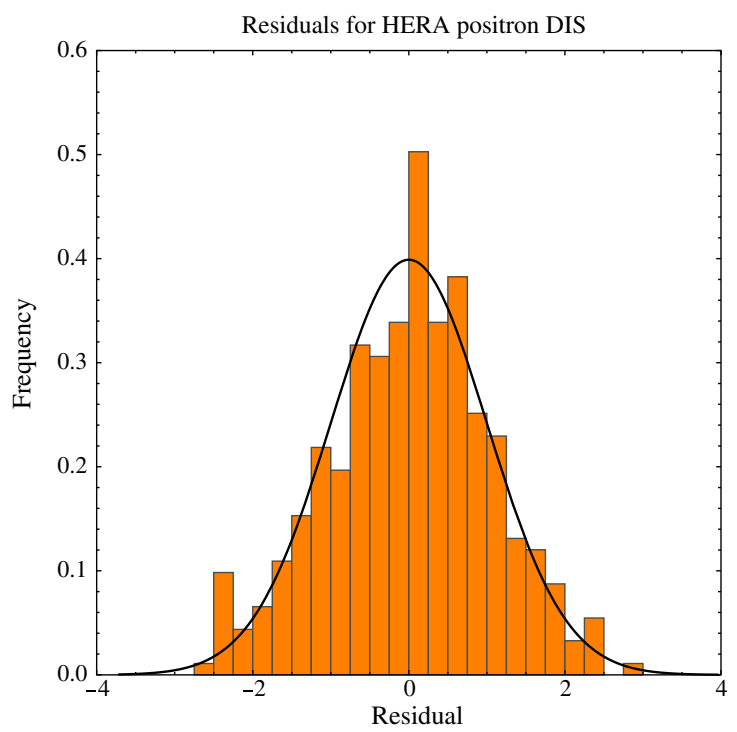

FIG. 9 (color online). Histogram of residuals for the HERA combined data for positron-proton NC DIS. The residuals are defined in Eq. (26). The solid curve is the standard normal distribution, for comparison.

histograms appear to be reasonably consistent with the superimposed standard normal distribution, within the limitations of the small number of data points in each $y_{j}$ bin.

We also check the size of the optimal shift parameters $\left\{\bar{\lambda}_{\alpha} ; \alpha=1,2,3, \ldots, 23\right\}$ and find that the histogram of the parameters shown in Fig. 14 is also consistent with the standard normal distribution. According to the figure, no

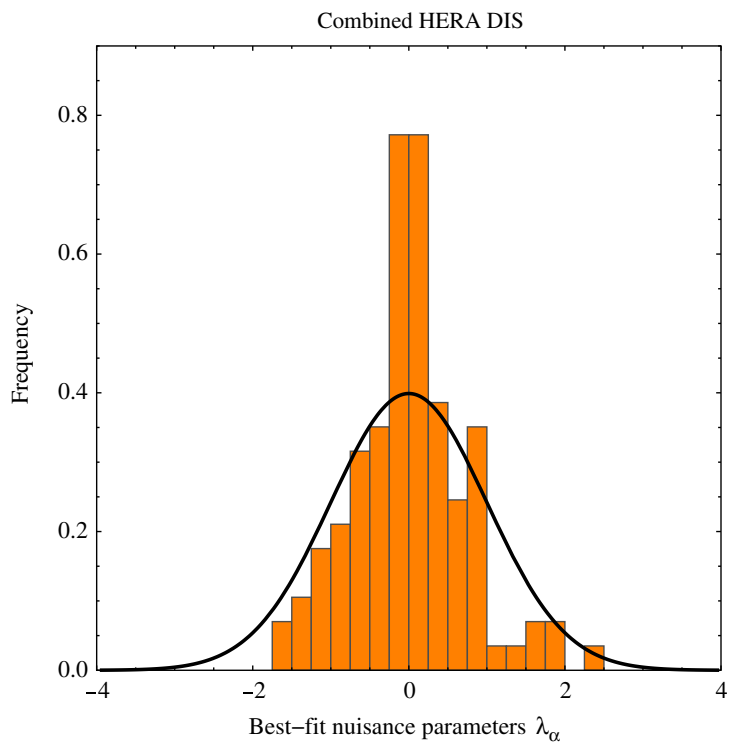

FIG. 10 (color online). Histogram of the optimal normalized shift variables $\left\{\bar{\lambda}_{\alpha}\right\}$ for the HERA combined data. There are 114 systematic errors, all of which apply to all of the 579 measurements in the HERA combined data set. The solid curve is the standard normal distribution, for comparison.

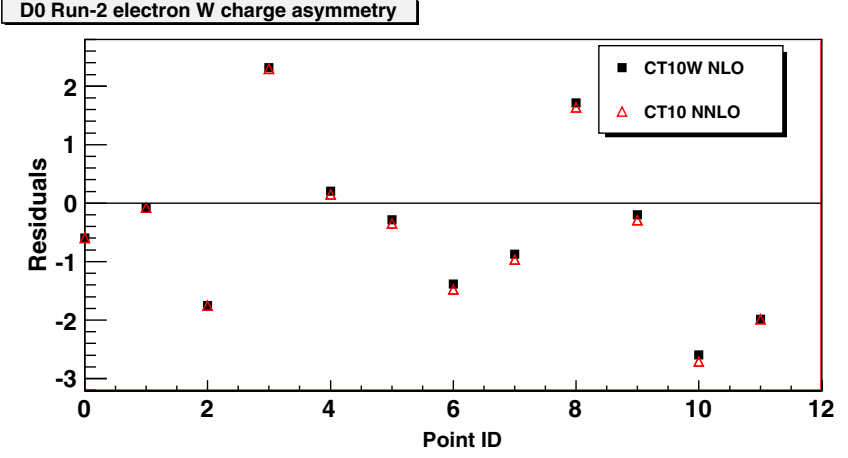

D0 Run-2 muon W charge asymmetry

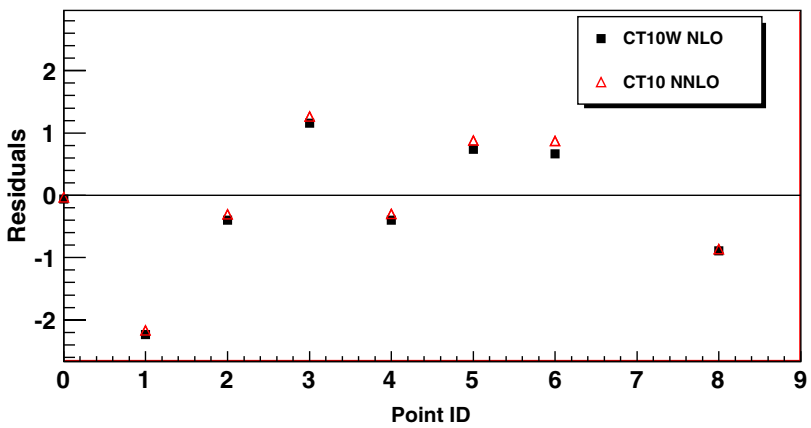

FIG. 11 (color online). Point-by-point residuals for the $\mathrm{D} \emptyset$ Run-2 charge asymmetry data in the electron (upper inset) and muon (lower inset) channels. The residuals are defined in Eq. (26).

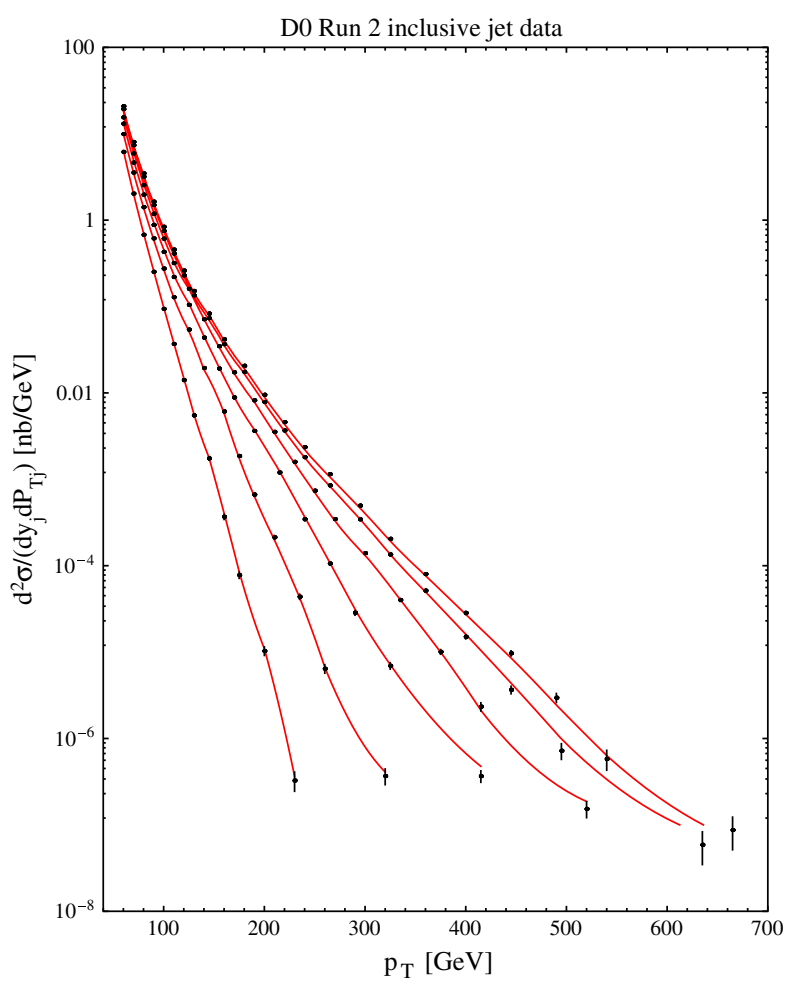

FIG. 12 (color online). Data and theory for the inclusive jet production data at $\mathrm{D} \emptyset$ Tevatron Run-2. The black points represent central values of the data, error bars are uncorrelated errors, and red curves are NLO theory obtained by using CT10NNLO PDFs. 

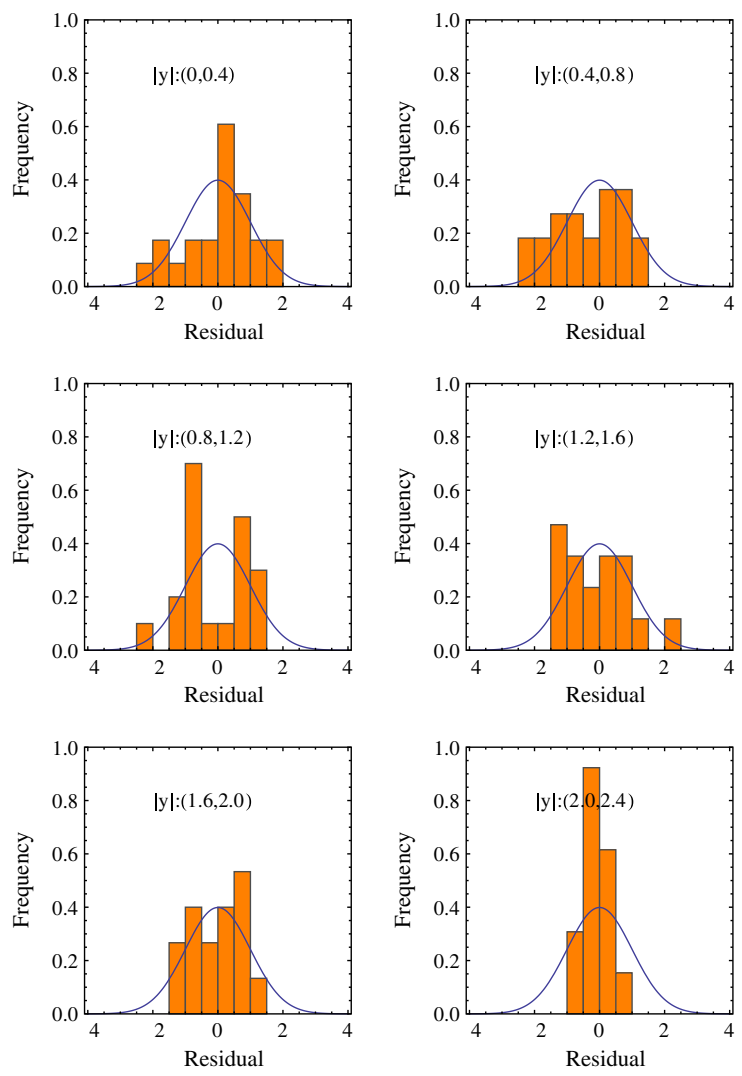

FIG. 13 (color online). Histograms of residuals for the DØ Tevatron Run-2 inclusive jet production data. Residuals are defined in Eq. (26). The solid curve is the standard normal distribution, for comparison.

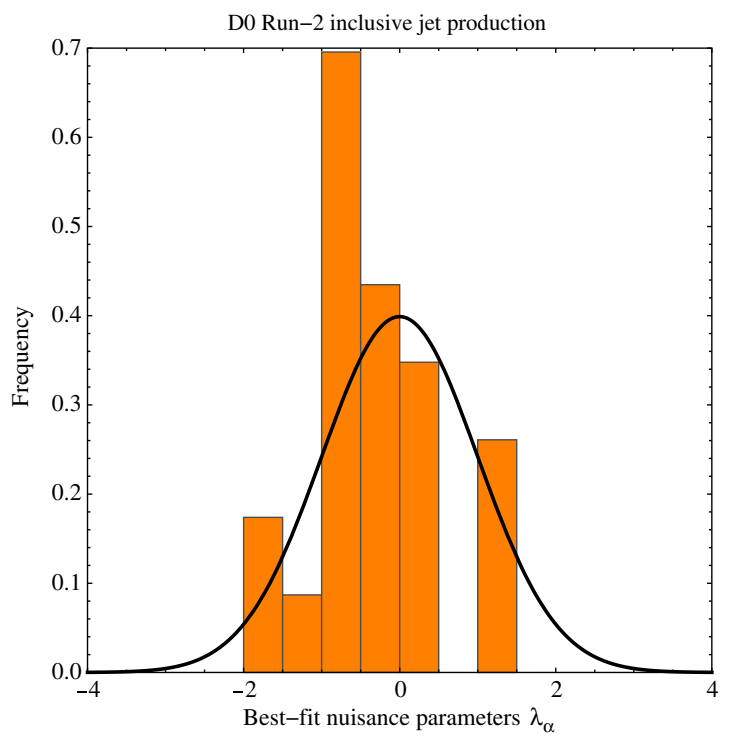

FIG. 14 (color online). Histogram of the optimal systematic shifts $\left\{\bar{\lambda}_{\alpha}\right\}$ for the $\mathrm{D} \emptyset$ Tevatron Run-2 inclusive jet production data.

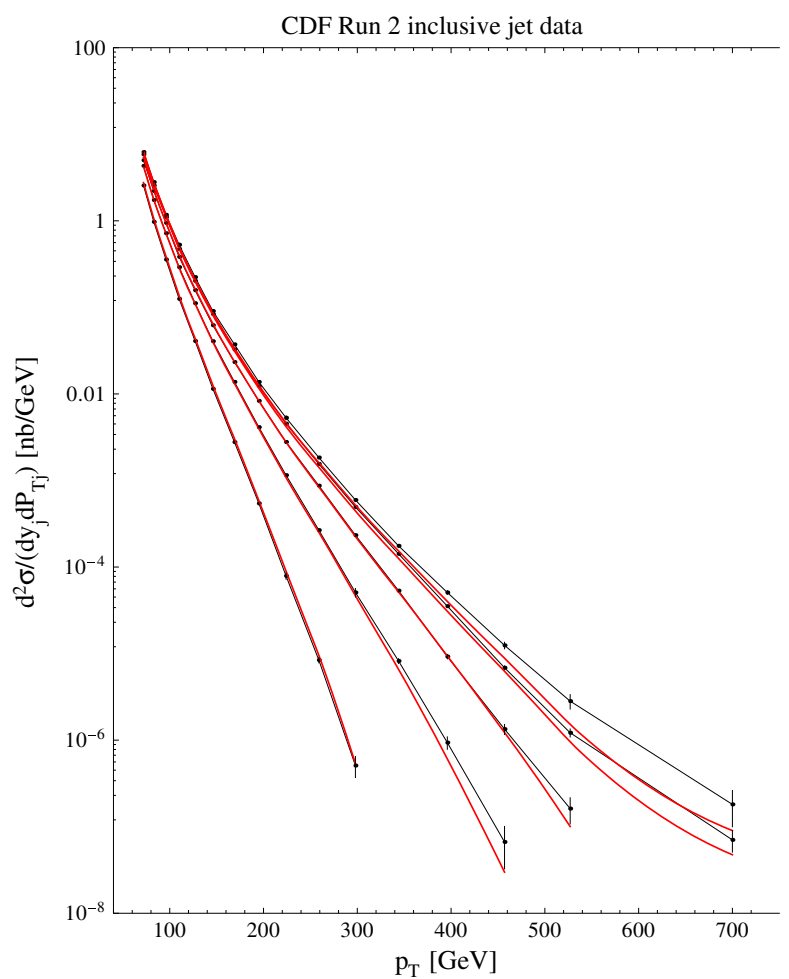

FIG. 15 (color online). Data and theory for inclusive jet production at CDF Tevatron Run-2. The black points are the central values of the data, the error bars are uncorrelated errors, and the red curves represent the NLO predictions computed by using CT10NNLO PDFs.

unreasonable shifts are required to create agreement between the theory and the data. Some of the systematic errors have a larger impact on the jet cross section than others, such as those related to the jet energy scale uncertainties.

A comparable measurement of inclusive jet production was performed by the CDF Collaboration in Run-2 [86]. This jet measurement has different systematic uncertainties than D0, and hence provides an independent constraint on the PDF parameters. A similar investigation indicates that the CT10 PDFs agree well with the CDF measurement. Figure 15 compares the $\mathrm{CDF}$ data set for inclusive jet production and the CT10 prediction, and the corresponding histogram of the residuals is shown in Fig. 16. The CDF value of $\chi_{E}^{2} / N_{p t}=102 / 72=1.42$ is higher than for $\mathrm{D} \emptyset$, but the histograms of the residuals do not reveal pronounced systematic disagreements. The contribution of 25 systematic shifts to the $\chi_{E}^{2}$ function of the CDF measurement is $\chi_{\lambda}^{2}=18$. The corresponding histogram of the optimal systematic shifts is included in Fig. 17.

\section{Correlated systematic errors in Tevatron jet production}

The results of the previous subsection show the importance of experimental systematic uncertainties in describing 

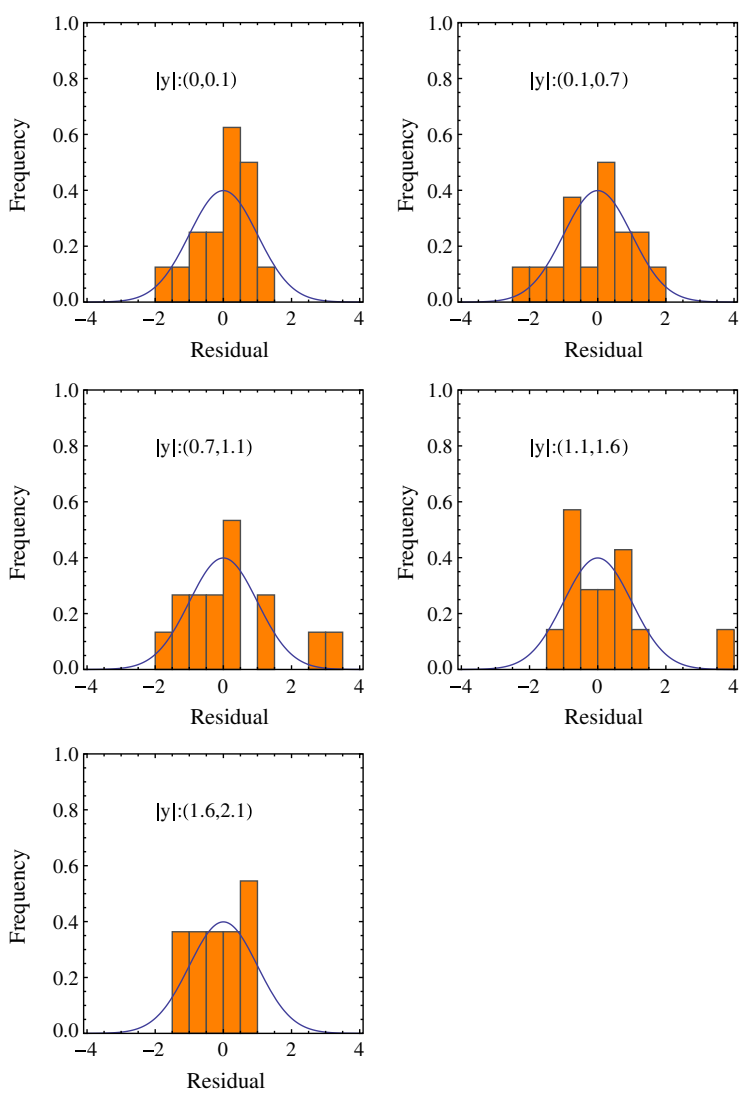

FIG. 16 (color online). Histogram of residuals [defined in Eq. (26)] for the CDF Run-2 inclusive jet production.

the Tevatron jet data. These uncertainties can be generally of two kinds, additive and multiplicative. For example, the underlying event and pileup uncertainties are additive errors. They are determined independently of the jet cross

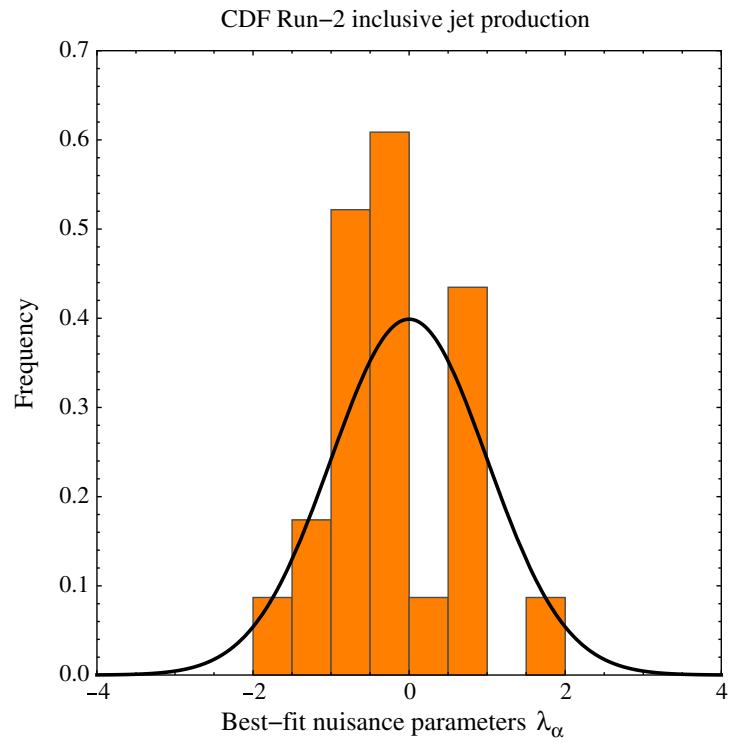

FIG. 17 (color online). Histogram of the optimal systematic shifts $\left\{\bar{\lambda}_{\alpha}\right\}$ for the CDF Tevatron Run-2 inclusive jet production data. section. The luminosity uncertainty and jet energy scale (JES) uncertainty are multiplicative errors. From the start, they are estimated as the percentage of the jet cross section in each bin. In jet production, there is no reason to handle the luminosity uncertainty differently from the JES and other multiplicative errors, as most of them have a comparable impact on the fit. ${ }^{9}$ Often, it is unknown if the error is additive or multiplicative. All of them are reconstructed from the published percentage values according to one of the methods that were reviewed in Sec. IIIC.

Both the QCD scale dependence of NLO jet cross sections and the detailed implementation of the systematic errors were explored when preparing our NNLO set of PDFs. An assortment of NNLO gluon PDF shapes, obtained by various treatments of jet production data in the global analysis and normalized to the shape of the CT10W NLO gluon PDF, is illustrated in Fig. 18. We compare five methods for the computation of $\beta_{i, \alpha}$ in jet production (cf. Sec. IIIC) and several choices of the renormalization and factorization scale. The CT10 central set and its PDF error band, both obtained by assuming method $D$ for computing $\beta_{i, \alpha}$ and the QCD scale $\mu=P_{T j}$, are shown by the solid black line and shaded green region, respectively, with $P_{T j}$ being the transverse momentum of an individual jet in each $P_{T}$ bin. For data sets other than the jet data in the global fit, we consistently use the extended $T$ definition of $\beta_{i, \alpha}$.

If the Run-2 jet cross sections are not included in the calculation of the global $\chi^{2}$ (or included with a small weight, such as 0.01), the fit generally prefers a softer gluon PDF at $x>0.1$, as indicated by the magenta long-dashed line in the figure. If $\alpha_{s}\left(M_{Z}\right)$ is fitted as well, its NNLO value in a fit without the jet data sets is also low, of order 0.114 .

With the jet data included, there is a general preference for an enhanced $g(x, Q)$ at large $x$, with some possible outcomes shown in Fig. 18. The curves were obtained using the extended $T, T$, and $D$ methods, for the factorization scales $\mu=P_{T j} / 2, P_{T j}, 2 P_{T j}$. Both the original method $T$ (applied only to the luminosity error) and extended method $T$ (applied to all correlated errors) produce a robust increase in $g(x, Q)$ at $x>0.2$, but the shape and magnitude of the enhancement vary with $\mu$. The $D$ method (black solid curve) results in a softer shape of the best-fit $g(x, Q)$, which lies between the fits with and without the jet data. All these curves lie well within the PDF error band of the public CT10 NNLO set.

\footnotetext{
${ }^{9}$ In the CTEQ6.X series and some other previous fits, the normalization of each experiment was fitted as a separate free parameter with a $\chi^{2}$ penalty imposed on its deviation from the nominal value. The procedure used was similar to the "normalization penalty trick" discussed in Ref. [95]. In the CT10 fits, the normalization uncertainty is instead included in the correlation matrix $\beta_{i, \alpha}$ and treated similarly to other multiplicative errors.
} 


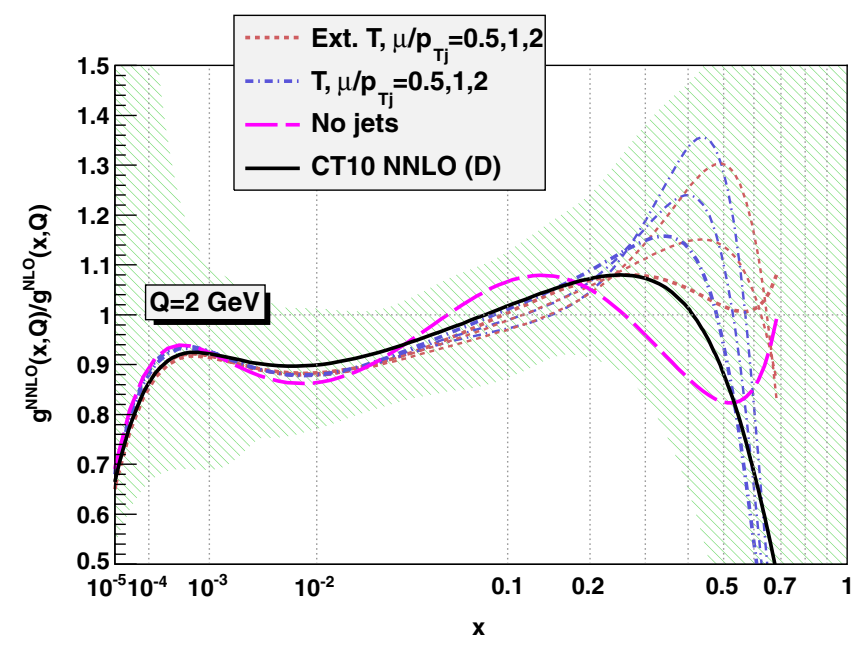

FIG. 18 (color online). Comparison of NNLO gluon PDF parametrizations obtained using different procedures for including jet production data sets in the global fit.

$\mathrm{g}(\mathrm{x}, \mathrm{Q}=85 \mathrm{GeV})$ normalized to CT10 NNLO

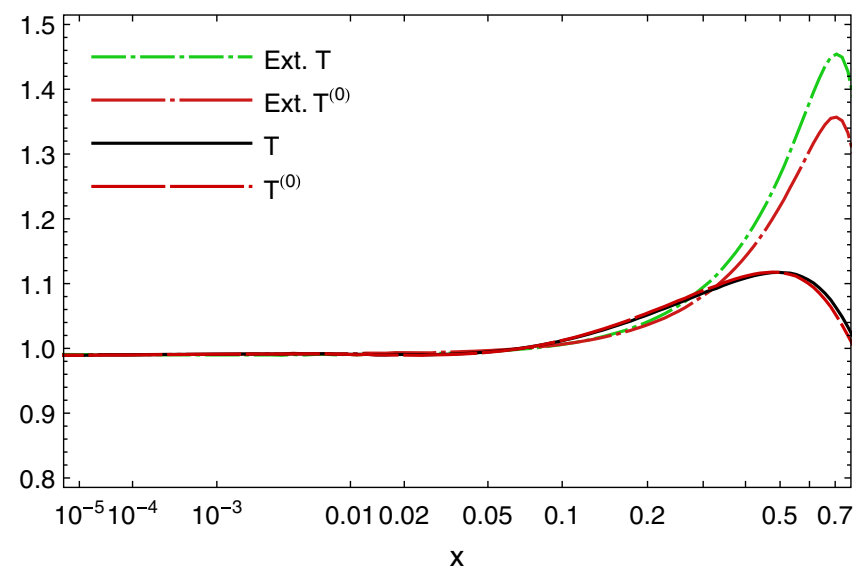

FIG. 19 (color online). Dependence of the gluon PDF on various definitions of the correlation matrices $\beta_{i \alpha}$ for the Tevatron Run 2 jet data in the global fit.

A complementary perspective is provided by Fig. 19, comparing the gluon $\operatorname{PDF} g(x, Q)$ at $Q=85 \mathrm{GeV}$ obtained with the five methods for computation of $\beta_{i \alpha}$ and the same factorization scale $\mu=P_{T j}$ in FASTNLO jet cross sections. All the curves are normalized to the CT10 NNLO prediction (based on the $D$ method). Here, we observe some differences between the (extended) $T$ and $T^{(0)}$ prescriptions. The extended $T$ method results in a drastic enhancement of $g(x, Q)$ at $x>0.3$, which is tempered when using the extended $T^{(0)}$ method. The $T$ and $T^{(0)}$ methods produce a stronger enhancement at $0.05<x<0.3$, but are flatter for $x>0.3$.

In all of the listed fits to the jet data, the quality of the fits are generally comparable. Table II shows representative output quantities for five methods: $\chi^{2} / N_{p t}$ for the CDF Run-2 and D0 Run-2 jet data sets, the best-fit luminosity parameter $\lambda_{\text {lumi }}$, and the best-fit $\alpha_{s}\left(M_{Z}\right)$ (if fitted). In all fits, the values of $\chi^{2} / N_{p t}$ are similar, with the $T / T^{(0)}$ methods showing a marginally better agreement with the CDF jet data $\left(\chi^{2} / N_{p t} \approx 1.23\right.$ vs $\left.1.4-1.5\right)$. If the QCD coupling strength is fixed in the fit to be $\alpha_{s}\left(M_{Z}\right)=0.118$, all luminosity shifts do not exceed 1.5 standard deviations; see the lines referring to "PDF only" fits.

However, if $\alpha_{s}\left(M_{Z}\right)$ is also fitted (the "PDF $+\alpha_{s}$ " fits), in the $D$ method we obtain a low $\alpha_{s}\left(M_{Z}\right)$ of 0.1137 , accompanied by a large luminosity shift $\bar{\lambda}_{\text {lumi }} \approx 2.7$. The $D$ method also results in a low $\chi^{2} / N_{p t}$ combination of 1.33 and 0.95 .

With four other methods, we obtain higher best-fit values of $\alpha_{s}\left(M_{Z}\right) \approx 0.116$, higher $\chi^{2}$ values, and the luminosity shifts below $2 \sigma$. This peculiarity of the "PDF $+\alpha_{s}$ " fit employing the $D$ method is suggestive of a marked D'Agostini bias. It does not occur at the same level in the "PDF only" fits that use a fixed $\alpha_{s}\left(M_{Z}\right)$, including CT10 NNLO.

Figure 20 compares the CT10 NNLO uncertainty band for the gluon PDF [normalized to the CT10W NLO $g(x, Q)]$ to the central NNLO gluon PDF parametrizations from ABM11, HERAPDF1.5, MSTW, and NNPDF2.3, evaluated with the same $\alpha_{s}\left(M_{Z}\right)=0.118$ as in CT10 NNLO. With the exception of ABM11, the central PDFs by other groups agree with CT10 NNLO within the 90\% C.L. uncertainty. The ABM11 gluon is distinctly smaller at $x>0.2$ and larger at $0.005<x<0.1$, most likely because of a different heavy-quark scheme (FFN) that the ABM11 NNLO set uses and its preference for a low $\alpha_{s}\left(M_{Z}\right)=0.1135$ that causes modifications in the preferred gluon shape $[51,52,94]$.

As can be seen from Fig. 18, by using method $D$ to determine the published CT10 NNLO set and the QCD scale $\mu=P_{T j}$, we obtain a PDF uncertainty band that also covers the gluon PDF parametrizations found with the alternative methods for the correlation matrix and other choices of QCD scales. The softer gluon behavior than in CT10W NLO is consistent with the preference of the nonjet experiments and results in lower $\chi^{2}$ for the combined HERA DIS set. It may also simulate the anticipated effect of missing NNLO corrections on the jet cross sections. While the method $T$ is generally preferable for the normalization of multiplicative normalization errors, and before additional recommendations on the separation of additive and multiplicative errors in inclusive jet measurements are provided by experimentalists, the uncertainty band for $g(x, Q)$ in Fig. 18 represents the current level of uncertainty in predicting the inclusive jet cross sections, due to variations in the fit procedure. These variations are typically smaller than the PDF error determined by the Hessian method. 
TABLE II. Best-fit values of $\chi_{E}^{2} / N_{p t}$ and the luminosity nuisance parameter $\lambda_{\text {lumi }}$ for the Tevatron Run-2 inclusive jet production, and for $\alpha_{s}\left(m_{Z}\right)$ in a fit with floating $\alpha_{s}\left(M_{Z}\right)$. The columns are obtained with the five methods for including correlated systematic errors in jet production. The first (second) numbers in each column correspond to the CDF (DØ) data sets, respectively.

\begin{tabular}{|c|c|c|c|c|c|c|}
\hline \multirow[b]{2}{*}{ Parameter } & \multirow[b]{2}{*}{ Type of the fit } & \multicolumn{5}{|c|}{ Method for computing $\beta_{i, \alpha}$} \\
\hline & & $D$ & $T$ & $T^{(0)}$ & Ext. $T$ & Ext. $T^{(0)}$ \\
\hline \multirow{4}{*}{$\begin{array}{l}\bar{\chi}_{E}^{2} / N_{p t} \text { for CDF }\left(N_{p t}=72\right) \\
\quad \text { and D0 }\left(N_{p t}=110\right) \\
\bar{\lambda}_{\text {lumi }}\end{array}$} & PDF only & $1.42,1.04$ & $1.23,1.05$ & $1.21,1.04$ & $1.48,1.03$ & $1.49,1.03$ \\
\hline & $\mathrm{PDF}+\alpha_{s}$ & $1.33,0.95$ & $1.24,1.07$ & $1.23,1.06$ & $1.48,1.06$ & $1.51,1.08$ \\
\hline & PDF only & $1.2,1.3$ & $-1.4,-1.2$ & $-1.5,-1.3$ & $0.53,0.90$ & $0.61,0.96$ \\
\hline & $\mathrm{PDF}+\alpha_{s}$ & $2.7,2.8$ & $-0.71,-0.51$ & $-0.75,-0.55$ & $1.2,1.5$ & $1.4,1.8$ \\
\hline \multirow[t]{2}{*}{$\bar{\alpha}_{s}\left(M_{Z}\right)$} & PDF only & 0.118 & 0.118 & 0.118 & 0.118 & 0.118 \\
\hline & $\mathrm{PDF}+\alpha_{s}$ & 0.1137 & 0.1159 & 0.1159 & 0.1162 & 0.1159 \\
\hline
\end{tabular}

\section{COLLIDER PHENOMENOLOGICAL PREDICTIONS}

A number of standard model cross section measurements from the LHC have been published, at center-of-mass energies of 7 and $8 \mathrm{TeV}$. Differential distributions are available from the $7 \mathrm{TeV}$ data (taken in 2010 and 2011), while the current $8 \mathrm{TeV}$ results (2012) are mostly in the form of inclusive cross section measurements. These data sets have not been used in the determination of the CT10 PDFs, but will be used in future fits.

An extensive comparison of theoretical predictions based on CT10NNLO and other PDF sets to a variety of LHC cross sections (for production of electroweak gauge bosons, inclusive jets, and top pairs) has been recently presented in [94]. We refer the reader to that paper for detailed comparisons of PDFs and parton luminosities by ABM, CT, HERAPDF, MSTW, and NNPDF groups; comparisons of theoretical predictions based on these PDFs and the LHC data; and the juxtaposition of the current experimental and theoretical uncertainties with the goal to quantify

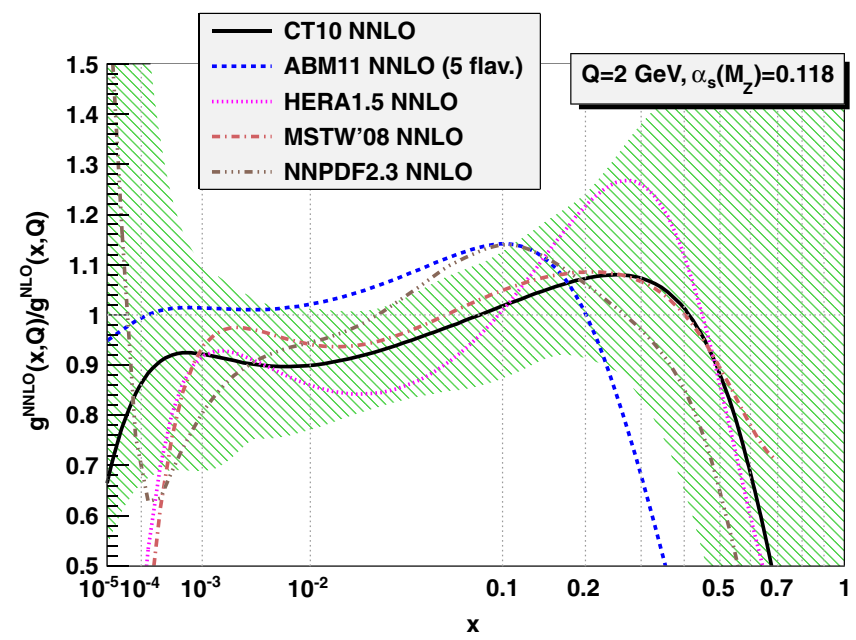

FIG. 20 (color online). Comparison of NNLO gluon PDF parametrizations obtained by different groups at the QCD scale $Q=2 \mathrm{GeV}$ and for $\alpha_{s}\left(M_{Z}\right)=0.118$. projections for constraining the PDFs in near-future LHC measurements.

In this section, we collect several comparisons of theoretical predictions for the LHC based on CT10 NNLO that support the discussion in the other sections and complement the benchmark comparisons in Ref. [94]. We focus on the predictions for LHC total cross sections, vector boson production, and inclusive jet production that will likely be included in the upcoming PDF fits. As a variety of comparisons with different PDF sets are published by the experimental groups, we include only select comparisons to illustrate general trends. In some figures, the CT10 predictions are compared to those from NNPDF2.3 [25] and MSTW2008 [24] that follow a similar methodology of the global QCD fit. ${ }^{10}$ All calculations use the native value of $\alpha_{s}\left(m_{Z}\right)$ for the PDF set. The PDF uncertainties are shown at the 68\% C.L, and the $\alpha_{s}$ uncertainties are not considered.

The LHC standard model cross sections are calculated with the following settings.

(i) For electroweak gauge boson production, the total cross sections are computed at NNLO using the program FEWZ2.1 $[109,110]$ and the factorization scale $Q=M_{V}$. The differential cross sections and asymmetries with cuts on the transverse momenta of the leptons are computed with the code ResBos $[111,112]$ that realizes the NNLL resummation of logarithms at small transverse momentum. The settings of the RESBos computation and nonperturbative function are the same as in [113], including the hard QCD scale that is equal to $M_{V} / 2$. This calculation provides a close approximation to the NNLO resummed cross section. The needed two-loop coefficients are obtained from the $\mathcal{O}\left(\alpha_{s}^{2}\right)$ cross sections at large transverse momentum $[114,115]$ and by requiring that the RESBos total rate coincides with the inclusive NNLO rate [116] computed by the CANDIA code $[117,118]$.

\footnotetext{
${ }^{10}$ Comparisons to the ABM11 and HERAPDF1.5 predictions can be found in Ref. [94].
} 
(ii) The cross section for top quark pair production is computed by the TOP++ code (version 1.4) [119]. The factorization and renormalization scale is chosen as $Q=m_{t}$, the top quark mass is $m_{t}=172.5 \mathrm{GeV}$ [64], and the other settings are as in Ref. [107]. The calculation includes the exact NNLO corrections to the $q \bar{q} \rightarrow \bar{t} t$ production [120] and an approximate NNLO combined with NNLL threshold logarithms in the other channels. Hence, the calculation provides a close to exact NNLO prediction at the Tevatron, where the $q \bar{q}$ channel dominates, and an approximate NNLO prediction at the LHC, where top production takes place primarily from $g g$ initial states.

(iii) Higgs boson production cross sections in the gluongluon fusion channel are computed at NNLO using the HNNLO code [121,122]. The factorization and renormalization scale was taken to be $Q=m_{H}=125 \mathrm{GeV}$, consistent with the recommendation of the LHC Higgs cross section working group [123]. Higgs production in the bottom quark fusion channel is computed at NNLO using the BBH@NNLO v1.3 code [124].

(iv) LHC inclusive jet cross sections are computed at NLO with the FASTNLO code [102-104] and cross checked against the MEKS code in $[94,100]$. The central scale in these predictions is chosen to be $\mu=P_{T j}$, the transverse momentum of each jet.
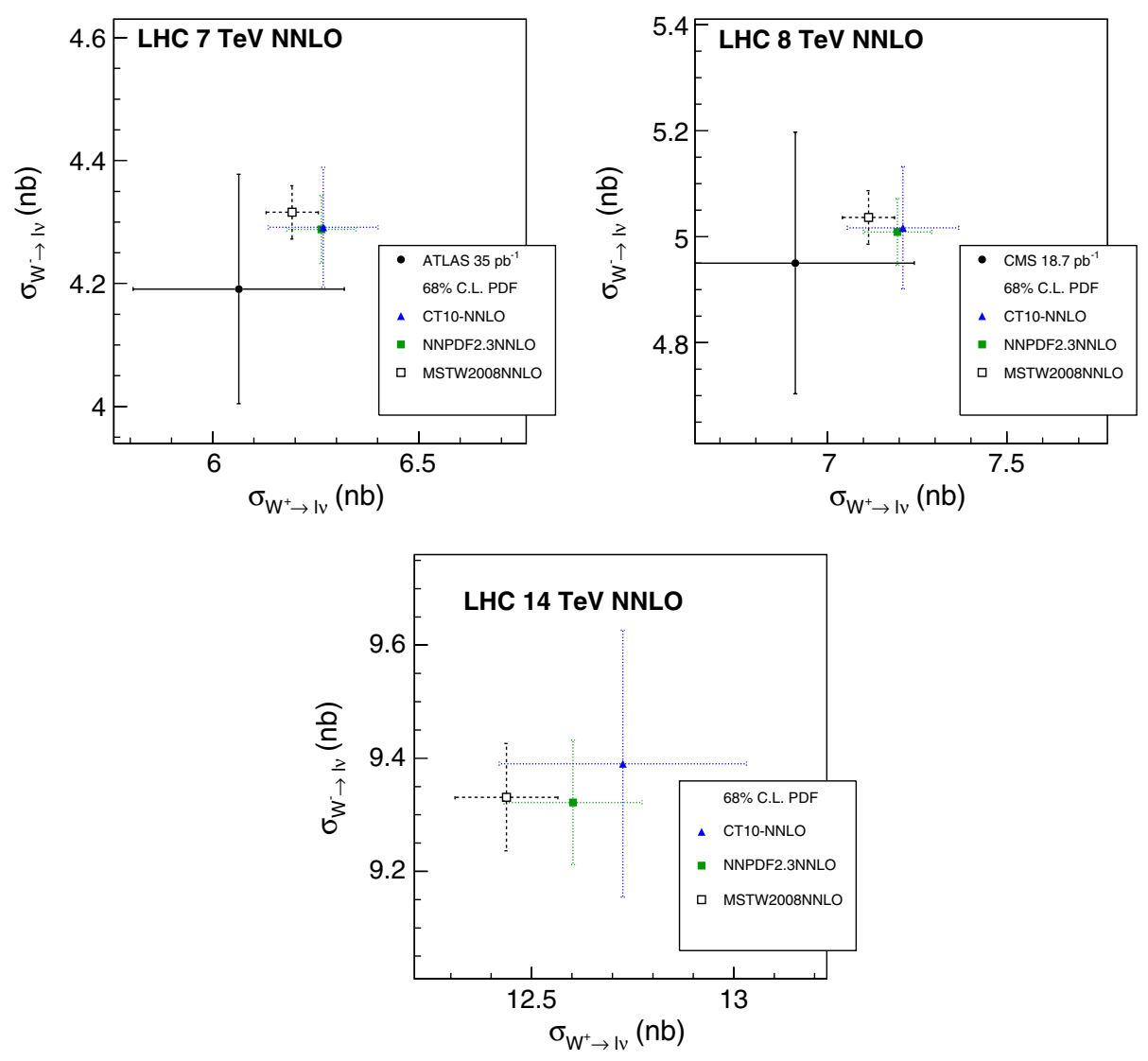

FIG. 21 (color online). NNLO $W^{+}$and $W^{-}$cross sections at the LHC. ellipses overlap well for all PDF sets. for the $W^{+}$and $W^{-}$comparisons.
In Fig. 23, we show the combined Tevatron total cross section for $t \bar{t}$ production [127] and ATLAS [128-132] and CMS [133] total cross sections, compared to approximate NNLO predictions. The experimental central values and uncertainty ranges are indicated by vertical lines and colored rectangles. For each collider energy, all cross production at 7,8 and $14 \mathrm{TeV}$, compared to ATLAS data TeV [125] and CMS data at $8 \mathrm{TeV}$ [126]. The CT10 and the predictions from the other NNLO PDFs listed. The central values of the data are below all NNLO predictions, but are within the uncertainties. For simplicity, the error bars for theoretical predictions indicate the symmetric PDF $W^{+}$and $W^{-}$are strongly correlated [4], but the $68 \%$ C.L. error

In Fig. 22, we show the NNLO predictions for the $W^{ \pm}$ and $Z$ total cross sections at 7,8 and $14 \mathrm{TeV}$, again $8 \mathrm{TeV}$ [126]. The conclusions are broadly similar to those

\title{
B. Top quark pair production, total cross sections
} compared to ATLAS data at $7 \mathrm{TeV}$ [125] and CMS data at

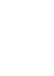



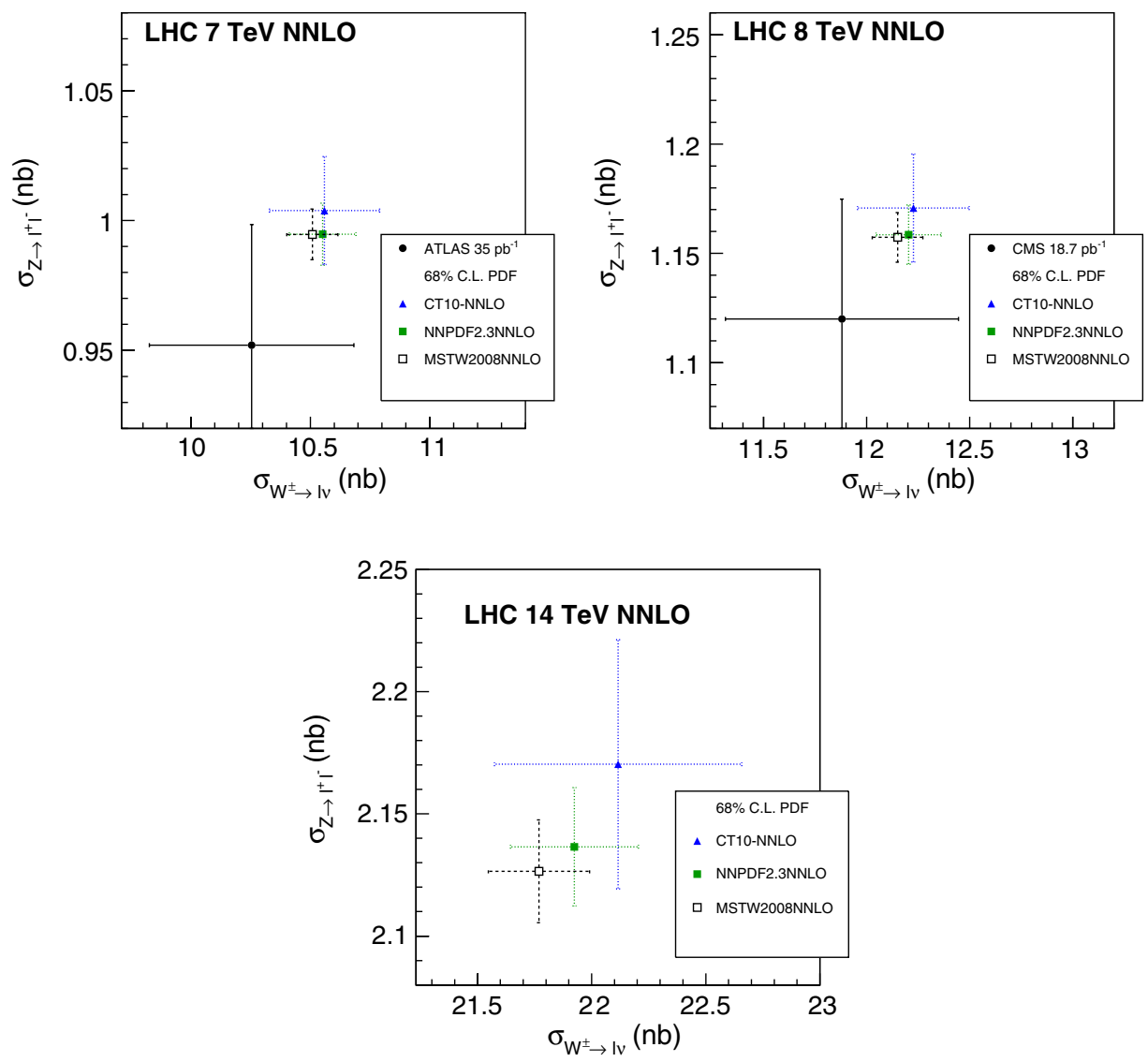

FIG. 22 (color online). NNLO $W^{ \pm}$and $Z$ cross sections at the LHC.

sections are normalized to the respective CT10NNLO prediction. Again, we observe good agreement between CT10NNLO and other theoretical predictions as well as the data.

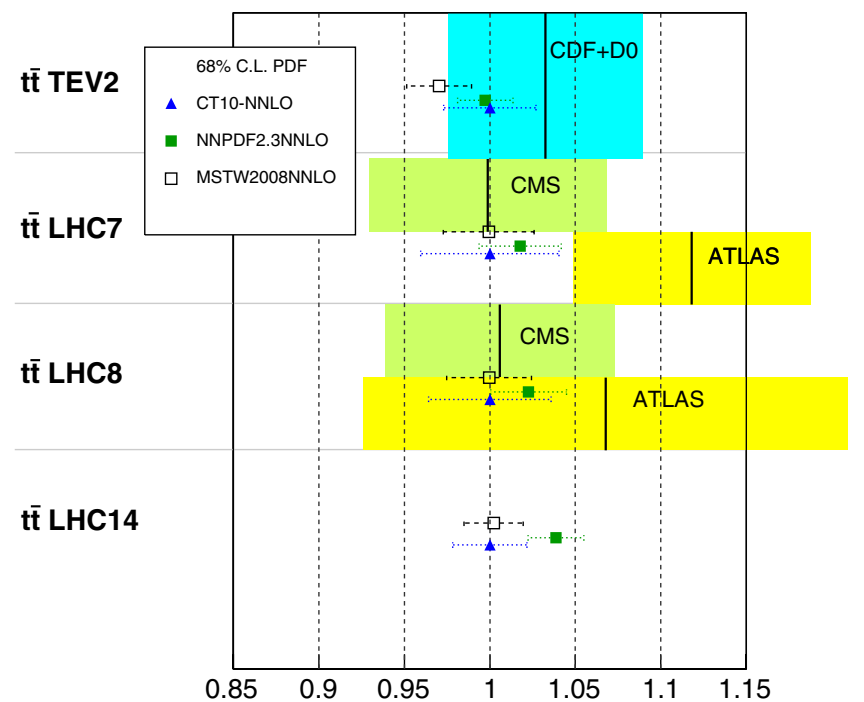

FIG. 23 (color online). Approximate NNLO predictions and experimental measurements of $t \bar{t}$ total cross sections at the Tevatron and LHC, normalized to CT10NNLO predictions.

\section{C. $W$ and $Z$ rapidity distributions}

More detailed PDF information can be gained by comparisons to $W / Z$ rapidity distributions and $W$ charge lepton asymmetry from ATLAS [125], CMS [134], and LHC-B [135] at $7 \mathrm{TeV}$. For differential observables, we use REsBos to account for multiple soft gluon radiation that may be non-negligible in the presence of constraints on the charged lepton's transverse momentum $p_{T \ell}$. Typical $W / Z$ production measurements require $p_{T \ell}$ to be above $20-25 \mathrm{GeV}$ in order to suppress charged leptons from background processes, and because of trigger requirements.

In some measurements, the lower $p_{T \ell}$ cut is as high as $35 \mathrm{GeV}$, approaching the position of the Jacobian peak in $d \sigma / d p_{T \ell}$ at $p_{T \ell} \approx M_{W} / 2 \approx 40 \mathrm{GeV}$ (for $W$ boson production), where large logarithms $\ln \left(Q_{T} / Q\right)$ dominate. ( $Q$ and $Q_{T}$ denote the invariant mass and transverse momentum of the weak boson, respectively.) With such high $p_{T \ell}$ cuts, the ResBos resummation calculation, which resums large logarithmic contributions to all orders in strong coupling, is expected to provide a better description of the data than the fixed-order calculations $[109,136,137]$. In practical comparisons, differences between the resummed and fixed-order NNLO calculations are most pronounced with an upper cut close to $p_{T \ell} \approx Q / 2$ [138]. The resummed and NNLO asymmetries are closer in general 

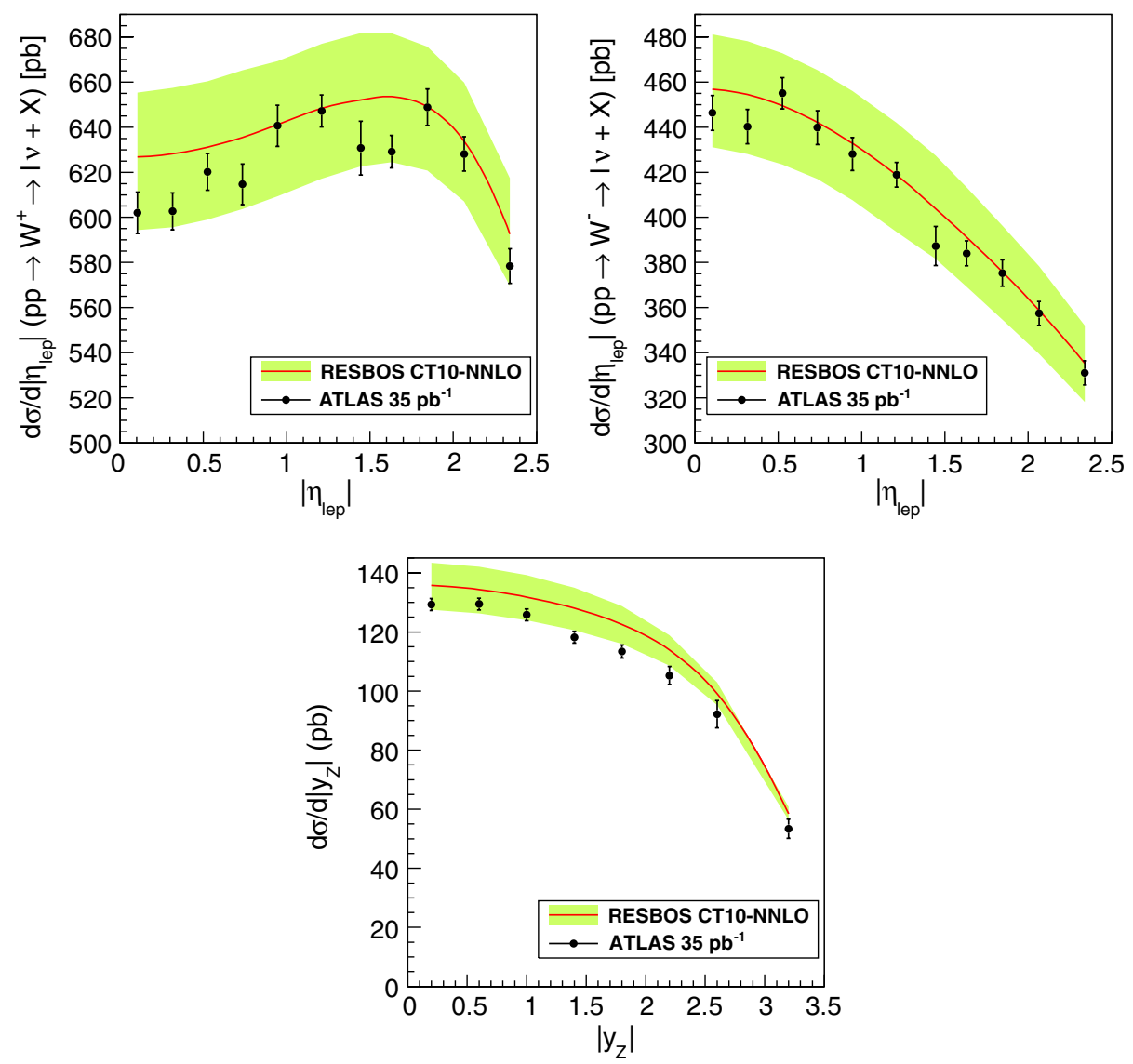

FIG. 24 (color online). Rapidity distributions of $Z$ and $W^{ \pm}$cross sections in ATLAS (35 $\left.\mathrm{pb}^{-1}\right)$ measurements at 7 TeV.

when only a lower cut is imposed. For instance, the REsBos and FEWZ 3.0 for the latest CMS $W$ asymmetry agree in both the $p_{T}^{\ell}>25$ and $35 \mathrm{GeV}$ bins with the FEWZ's theoretical uncertainty [139].

In Fig. 24 we show a comparison between predictions for the $Z$ and $W^{ \pm}$lepton rapidity distributions and the data measured by the ATLAS Collaboration using $35 \mathrm{pb}^{-1}$ of integrated luminosity at $7 \mathrm{TeV}$ [125]. In all three figures the green band represents the PDF uncertainty, while the red solid line shows the central prediction. For the $Z$ rapidity distribution, the central RESBos prediction overshoots the data by a few percent, but is within the PDF uncertainties
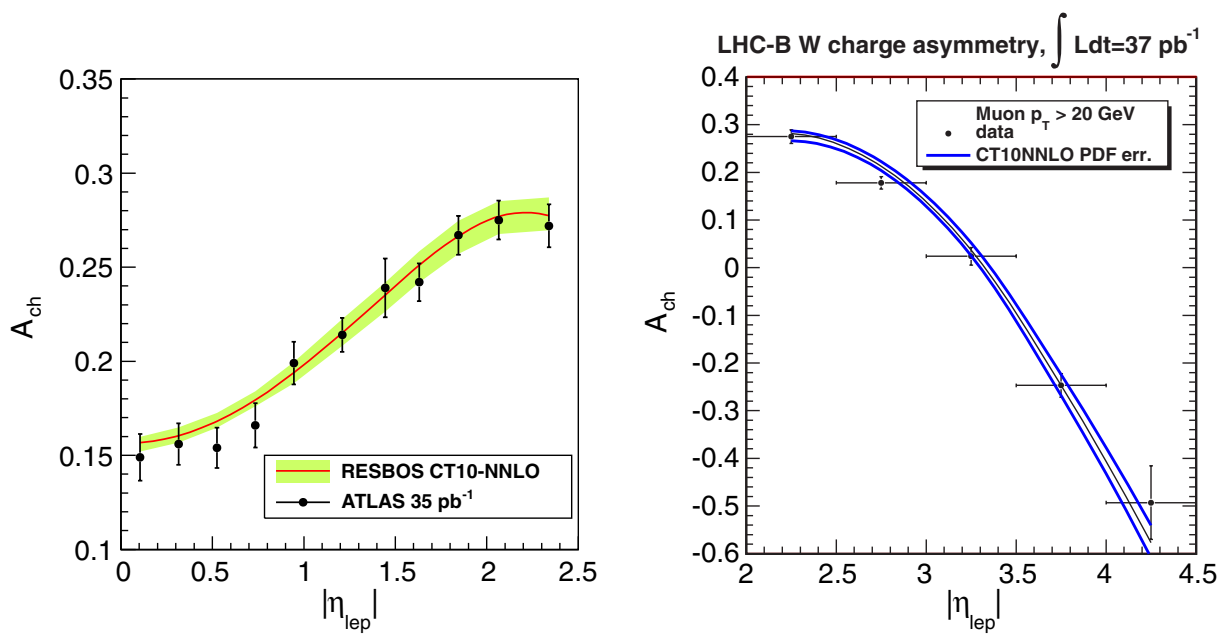

FIG. 25 (color online). A lepton rapidity distribution of the $W^{ \pm}$charge asymmetry in the ATLAS $\left(35\right.$ pb $\left.^{-1}\right)$ measurement $(l e f t)$ and LHC-B $\left(37 \mathrm{pb}^{-1}\right)$ measurement (right) at $7 \mathrm{TeV}$. 
from the data. These $\mathrm{LHC} Z / W^{ \pm}$data are yet to be included in the fits and may modify the quark PDFs at small $x$. It is interesting to note that ATLAS found that the tight constraints on the relevant normalization between the $W$ and $Z$ data sets resulted in a larger-than-expected strange quark density in the $x$ range represented by the $Z$ and $W^{ \pm}$ lepton rapidity distributions [140]. We shall include these (and upcoming) data sets, with correlated systematic error analysis, in our next run of global analysis.

In Fig. 25, a ResBos prediction is compared to the lepton charge asymmetry from ATLAS and LHC-B [135] at $7 \mathrm{TeV}$ as a function of the lepton pseudorapidity $\eta_{\text {lep }}$. The agreement of CT10 NNLO with both asymmetry sets is good, e.g., $\chi_{E}^{2} / N_{p t}=0.45$ for ATLAS $W$ asymmetry. The LHC-B experiment probes a different kinematic range (more forward coverage) than either ATLAS or CMS, and thus can provide unique information on large- $x$ quark distributions. In this figure, the central prediction and PDF error are indicated by the black solid and blue solid lines, respectively.

The LHC $W$ charge asymmetry measurements have the potential to test some combinations of the PDFs that are still poorly constrained. For example, the measurement of the charge asymmetry in the rapidity bins of CMS in Fig. 26 probes valence quark PDFs, $u_{v}(x, Q) \equiv u(x, Q)-$ $\bar{u}(x, Q)$ and $d_{v}(x, Q) \equiv d(x, Q)-\bar{d}(x, Q)$ in the small- $x$ region that is not constrained by the previous data.

Figure 27 shows the PDF-induced correlation cosine $\cos \varphi[4,89]$ between the LHC $W$ charge asymmetry in several bins of lepton rapidity $\eta_{\text {lep }}$ and valence $u$ and $d$ quark PDFs and the ratio $d(x, Q) / u(x, Q)$ of the CT10NNLO PDF set. In the intervals of $x$ where $\cos \varphi$ is close to \pm 1 , the $W$ asymmetry data at the shown $\eta_{\text {lep }}$ values is sensitive to the PDF uncertainty on the indicated parton distributions. A strong correlation can be observed with $u_{v}$ at $0.01<x<0.15$ and $d_{v}$ at $0.005<x<0.05$. Both valence PDFs show a strong anticorrelation at $x<0.001$ as a consequence of the valence sum rules. The $d / u$ ratio shows a strong correlation at $0.0005<x<$ 0.005 and anticorrelation at $0.05<x<0.2$. The other PDF flavors are not strongly correlated with the $W$ asymmetry in these rapidity ranges.

The current CMS $W$ asymmetry data shown in the left panel of Fig. 26 agree reasonably with the CT10 NNLO PDFs, given the present PDF uncertainties and experimental uncertainties. The CMS data are slightly below the central CT10 prediction at $\left|\eta_{\text {lep }}\right|<1$, but within the PDF uncertainty band. The disagreement is increased at $\left|\eta_{\text {lep }}\right|<2$, where we also observe less regular behavior of the data.

We note that the reconstruction of the present CMS data has a systematic uncertainty associated with the discrimination from background processes. In the Tevatron $W$ asymmetry measurements, it was essential to require a sufficiently high missing $E_{T}$ (MET), of order $20 \mathrm{GeV}$ or more, to discriminate the $W$ boson decay events from the significant background. The MET requirement has not been imposed in the current CMS measurement, which applied a different technique to suppress the background.

In the ResBos theoretical calculation, we cannot exactly implement the experimental background subtraction technique when comparing to the CMS data. At the lowest order in perturbative QCD (when $Q_{T}=0$ ), the additional MET cut is not needed, as the condition $p_{T l}>35 \mathrm{GeV}$ automatically implies MET $>35 \mathrm{GeV}$. At higher orders, the $p_{T l}$ and MET cuts are no longer equivalent. The predictions for $W$ charge asymmetry depend on the assumed MET cut; cf. the right panel in Fig. 26. This behavior suggests that subtle effects in the separation of $W$ boson events from the background may be comparable to the observed differences between CT10 NNLO theory and CMS data in Fig. 26.

In the absence of applicable experimental constraints in the CT10 fit, the small- $x$ behavior of the $u_{v}$ and $d_{v}$ PDFs is
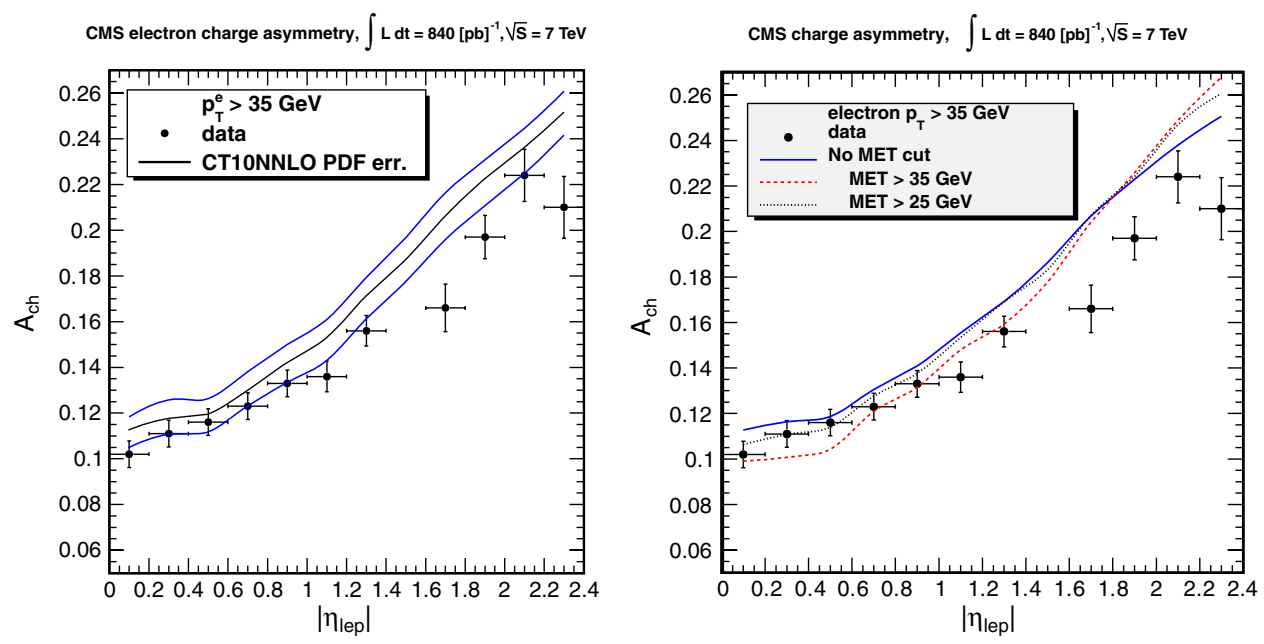

FIG. 26 (color online). A lepton rapidity distribution of the $W^{ \pm}$charge asymmetry in the CMS $\left(840 \mathrm{pb}^{-1}\right)$ measurement at 7 TeV. 


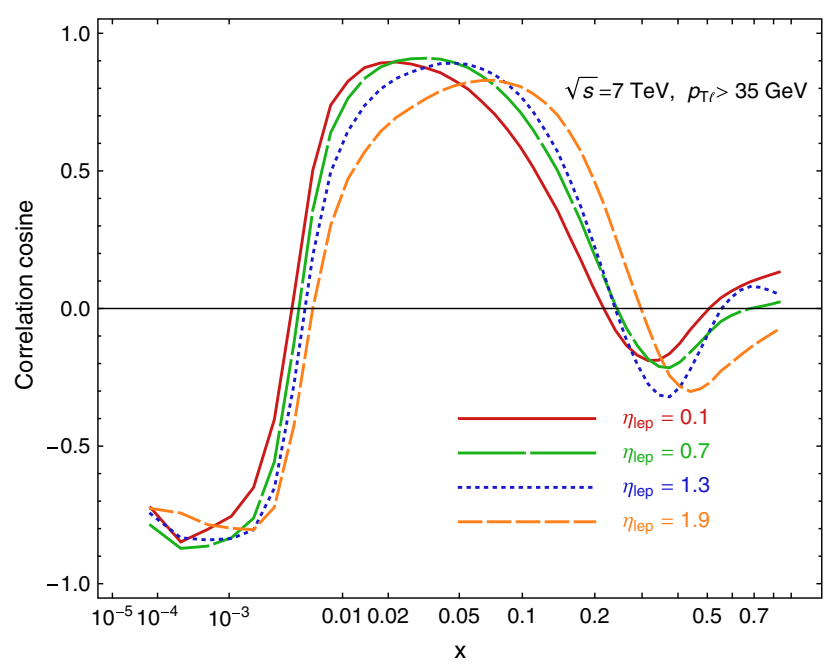

Correlation, $A_{\mathrm{ch}}\left(\eta_{\mathrm{lep}}\right)$ and $\mathrm{d}(\mathrm{x}, \mathrm{Q}) / \mathrm{u}(\mathrm{x}, \mathrm{Q})$ at $\mathrm{Q}=85$. $\mathrm{GeV}$

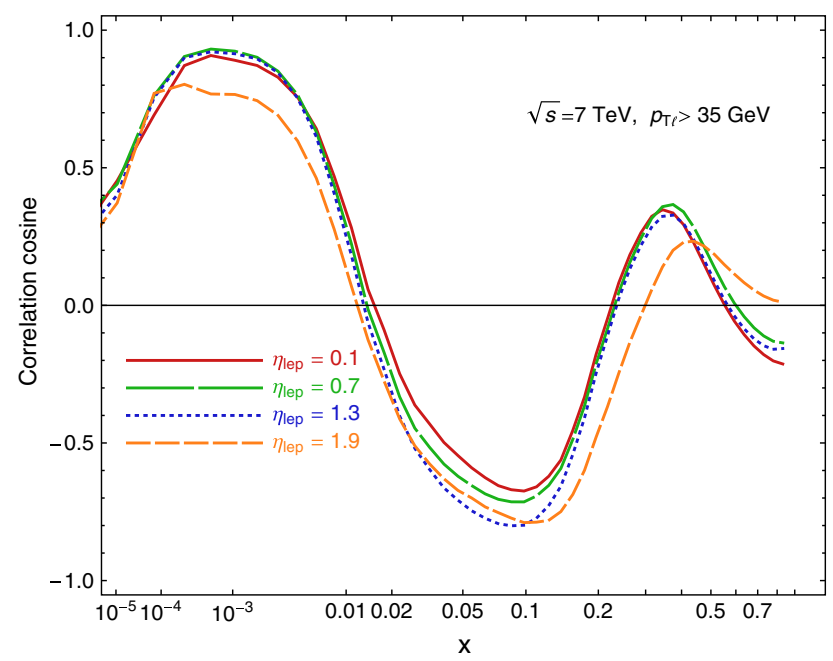

FIG. 27 (color online). PDF-induced correlations between $W$ charge asymmetry $A_{c h}\left(\eta_{\text {lep }}\right)$ at the LHC 7 TeV and $u_{v}(x, Q)=u(x, Q)-\bar{u}(x, Q), d_{v}(x, Q)=d(x, Q)-\bar{d}(x, Q)$, and $d(x, Q) / u(x, Q)$ at $Q=85 \mathrm{GeV}$. The charge asymmetry is evaluated at lepton rapidity values indicated in the figure.

assumed to be governed by a shared $x$ power, $u_{v}\left(x, Q_{0}\right)$, $d_{v}\left(x, Q_{0}\right) \sim x^{A_{1 v}}$ in the $x \rightarrow 0$ with the same $A_{1 v} \approx-1 / 2$. This Regge-inspired assumption partly explains the correlations of PDFs at small $x$. It will be tested by including the LHC $W$ asymmetry in the upcoming fits.

\section{ATLAS inclusive jet distribution}

In Figs. 28 and 29, we compare the inclusive cross section predictions to the ATLAS measurements using the anti- $k_{T}$ jet algorithm with $R=0.6$. In Fig. 28, the comparisons are made to the ATLAS raw, unshifted data; in Fig. 29, the optimal systematic shifts have been applied to the ATLAS central values. The scale uncertainty in the theory prediction is calculated by varying the QCD scale in the range $P_{T j} / 2 \leq \mu \leq 2 P_{T j}$. The uncertainties of the data points shown in Figs. 28 and 29 are evaluated by adding the statistical and uncorrelated systematic errors in quadrature.

The overall agreement of the shifted data with the (NLO) predictions utilizing CT10NNLO PDFs is good even without theory uncertainty: $\chi_{E}^{2} / N_{p t}=0.74(0.78)$ for $R=0.6(0.4)$. Some differences in the shape and normalization observed for the unshifted data disappear upon application of the systematic error shifts. The $\chi_{E}^{2}$ function is defined according to the extended $T$ convention for the ATLAS correlation matrix. Slightly higher values of $\chi_{E}^{2}$ would be obtained with alternative conventions for $\beta_{i, \alpha}$ [94].

\section{E. Higgs boson total cross sections in the standard model}

Now we turn to the NNLO total cross sections for Higgs boson production at both the Tevatron and LHC via 

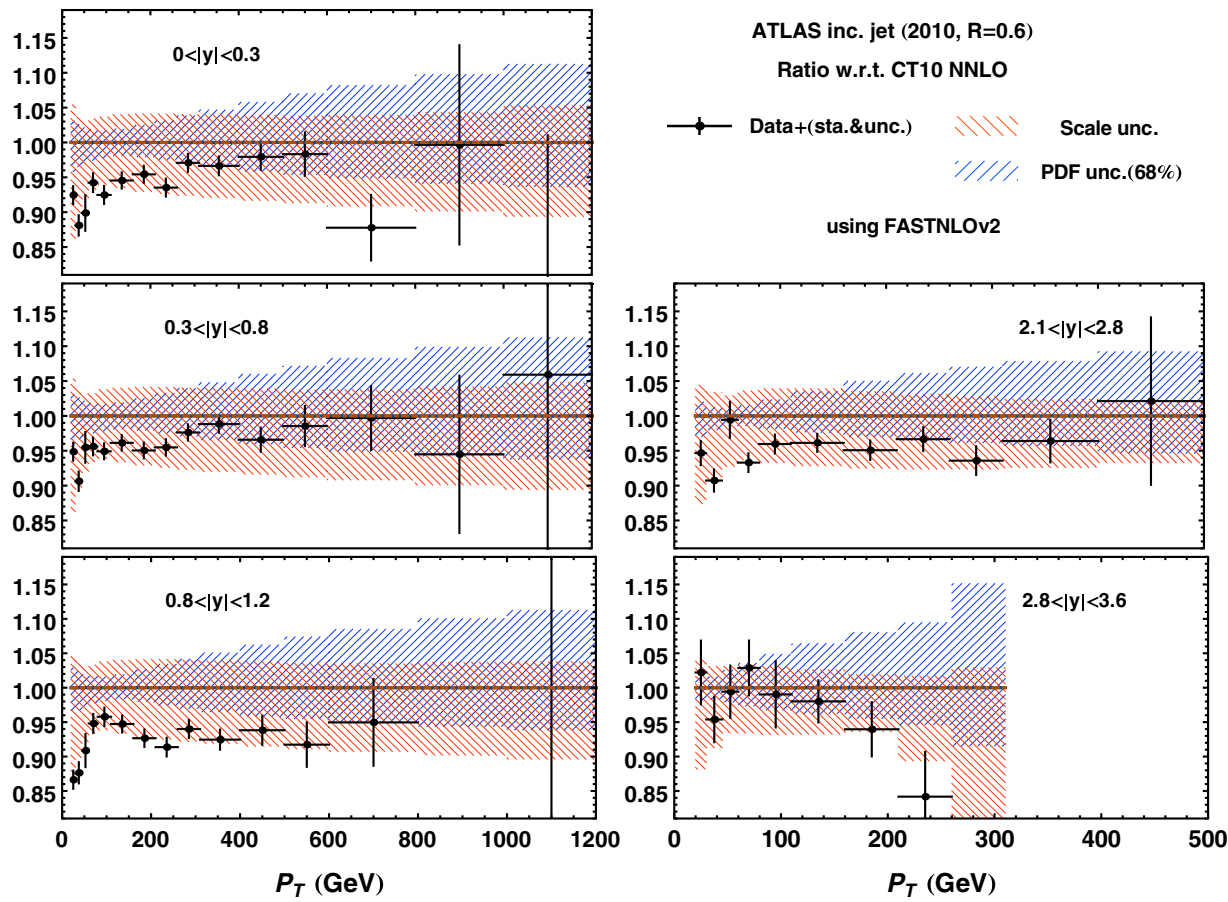

FIG. 28 (color online). Comparison of CT10NNLO predictions with unshifted 2010 ATLAS inclusive jet data $(R=0.6)$.

gluon-gluon fusion [121,122] and $b \bar{b}$ annihilation [124], shown as ratios to the CT10NNLO prediction in Fig. 30. At the Tevatron, PDF uncertainties of the Higgs boson production cross section via gluon-gluon fusion are large and cover all the central predictions. At the LHC, the CT10NNLO gluon-gluon fusion predictions are in a good agreement with those from the other PDF groups, except for NNPDF2.3 at 7 and $8 \mathrm{TeV}$, which predicts larger cross sections. For Higgs boson production via $b \bar{b}$ annihilation, the NNLO predictions obtained with all PDFs are consistent within the quoted PDF uncertainties.
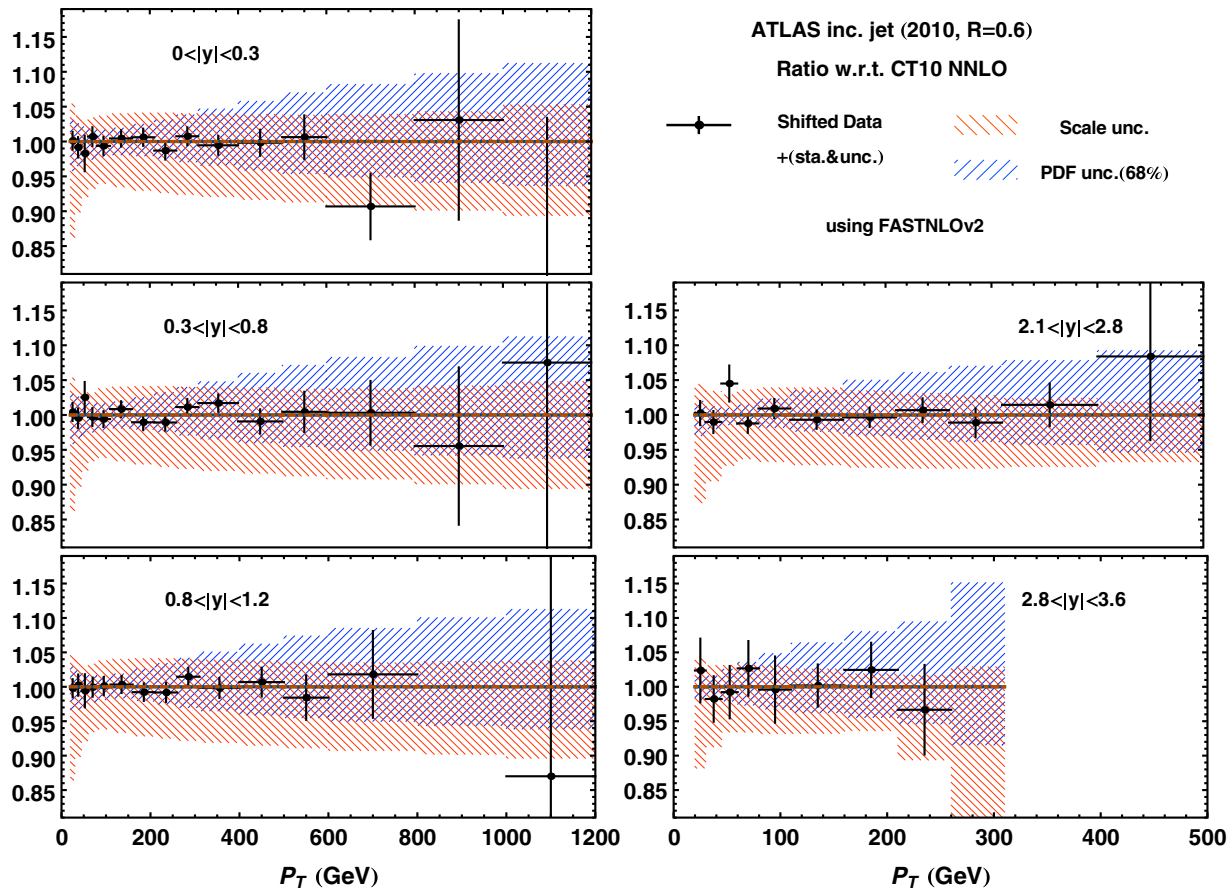

FIG. 29 (color online). Comparison of CT10NNLO predictions with shifted 2010 ATLAS inclusive jet data $(R=0.6)$. 

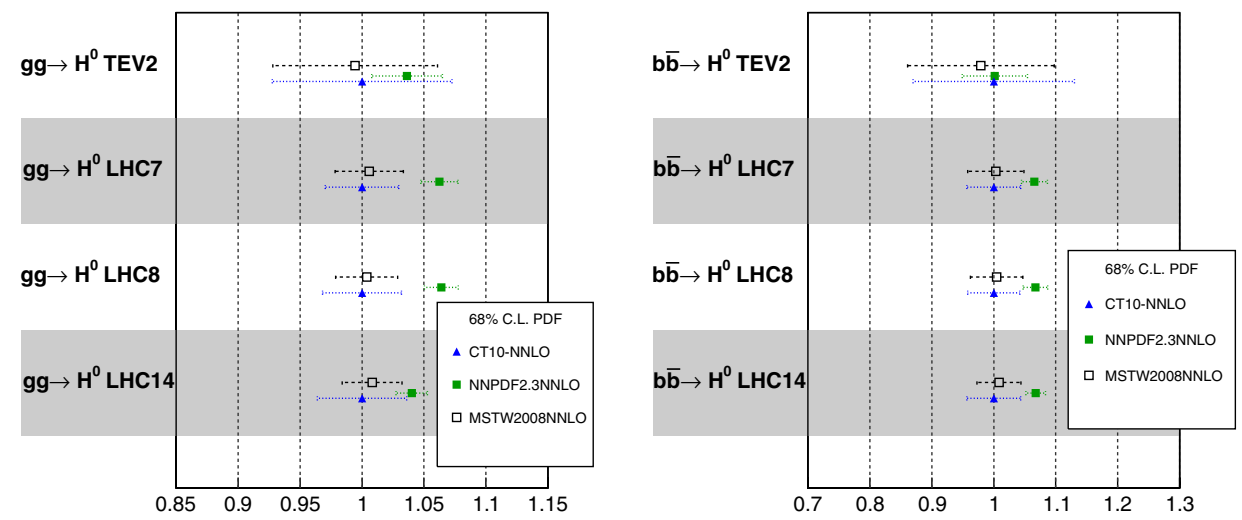

FIG. 30 (color online). NNLO cross sections of Higgs boson production via gluon fusion (left) and bottom quark annihilation (right), normalized to CT10NNLO predictions.

\section{DISCUSSION AND CONCLUSION}

We have presented NNLO PDFs from the CTEQ-TEA group. These CT10NNLO PDFs have been determined based on essentially the same global data sets used in the previous CT10 and CT10W NLO PDF analyses. In this new analysis, the effects of finite quark masses have been implemented in the S-ACOT- $\chi$ scheme at NNLO accuracy. We obtain a similar quality of agreement with the fitted experimental data sets in the NNLO fit as at NLO.

We find that at low $x$ (below $10^{-2}$ ), the NNLO gluon distribution is suppressed, while the quark distributions increase, compared to the same distributions at NLO. The $\mathcal{O}\left(\alpha_{s}^{2}\right)$ GM-VFN scheme used in the NNLO fit results in changes to the heavy quark distributions, both charm and bottom. The large- $x$ gluon and $d$-quark distributions are reduced due to (1) the removal of the Tevatron Run-1 inclusive jet data, (2) the alternate treatment of correlated systematic errors and choices of renormalization and factorization scales in jet cross sections, and (3) the revised electroweak couplings in DIS cross sections.

Compared to the MSTW2008 NNLO PDFs, the gluon and quark distributions are larger as $x$ approaches zero (the CT10 parametrization requires a positive gluon for $Q$ above
1.3 $\mathrm{GeV}$ ), while the strangeness distribution is larger over most of the $x$ range. The differences tend to decrease as $Q^{2}$ increases.

We have compared NNLO predictions using CT10 to available LHC data and have found good agreement. Numerical comparisons presented here complement a more detailed benchmark study of the dependence of LHC predictions on PDFs and $\alpha_{s}\left(M_{Z}\right)$ that has been recently released [94]. The available LHC data are already providing important information on PDFs, and future data will provide even stronger constraints. This will be developed further in a future publication.

\section{ACKNOWLEDGMENTS}

This work was supported by the U.S. DOE Early Career Research Award No. DE-SC0003870 and by LightnerSams Foundation; by the U.S. Department of Energy under Grant No. DE-FG02-96ER40969; by the U.S. National Science Foundation under Grant No. PHY-0855561; by the National Science Council of Taiwan under Grants No. NSC-98-2112-M-133-002-MY3 and No. NSC-1012112-M-133-001-MY3. The authors thank Gavin Salam for his help with the setup of the HOPPET code.
[1] J. Pumplin, D. R. Stump, J. Huston, H.-L. Lai, P. Nadolsky, and W.-K. Tung, J. High Energy Phys. 07 (2002) 012.

[2] D. Stump, J. Huston, J. Pumplin, W.-K. Tung, H.-L. Lai, S. Kuhlmann, and J. F. Owens, J. High Energy Phys. 10 (2003) 046.

[3] W.-K. Tung, H. L. Lai, A. Belyaev, J. Pumplin, D. Stump, and C.-P Yuan, J. High Energy Phys. 02 (2007) 053

[4] P. Nadolsky, H.-L. Lai, Q.-H. Cao, J. Huston, J. Pumplin, D. Stump, W.-K. Tung, and C.-P. Yuan, Phys. Rev. D 78, 013004 (2008).
[5] J. Pumplin, J. Huston, H. Lai, P. Nadolsky, W.-K. Tung, and C.-P. Yuan, Phys. Rev. D 80, 014019 (2009).

[6] M. Aivazis, J. C. Collins, F. I. Olness, and W.-K. Tung, Phys. Rev. D 50, 3102 (1994).

[7] J. C. Collins, Phys. Rev. D 58, 094002 (1998).

[8] M. Kramer, F. I. Olness, and D. E. Soper, Phys. Rev. D 62 , 096007 (2000).

[9] W.-K. Tung, S. Kretzer, and C. Schmidt, J. Phys. G 28, 983 (2002).

[10] H.-L. Lai, M. Guzzi, J. Huston, Z. Li, P. M. Nadolsky, J. Pumplin, and C.-P. Yuan, Phys. Rev. D 82, 074024 (2010). 
[11] S. Moch, J. Vermaseren, and A. Vogt, Nucl. Phys. B688, 101 (2004).

[12] A. Vogt, S. Moch, and J. Vermaseren, Nucl. Phys. B691, 129 (2004).

[13] J. Sanchez Guillen, J. Miramontes, M. Miramontes, G. Parente, and O. Sampayo, Nucl. Phys. B353, 337 (1991).

[14] W. van Neerven and E. Zijlstra, Phys. Lett. B 272, 127 (1991).

[15] E. Zijlstra and W. van Neerven, Phys. Lett. B 273, 476 (1991).

[16] E. Zijlstra and W. van Neerven, Nucl. Phys. B383, 525 (1992).

[17] E. Laenen, S. Riemersma, J. Smith, and W. van Neerven, Nucl. Phys. B392, 162 (1993).

[18] S. Riemersma, J. Smith, and W. van Neerven, Phys. Lett. B 347, 143 (1995).

[19] M. Buza, Y. Matiounine, J. Smith, R. Migneron, and W. van Neerven, Nucl. Phys. B472, 611 (1996).

[20] C. Anastasiou, L. J. Dixon, K. Melnikov, and F. Petriello, Phys. Rev. Lett. 91, 182002 (2003).

[21] C. Anastasiou, L. J. Dixon, K. Melnikov, and F. Petriello, Phys. Rev. D 69, 094008 (2004).

[22] A. Gehrmann-De Ridder, T. Gehrmann, E. Glover, and J. Pires, J. High Energy Phys. 02 (2013) 026.

[23] A. Gehrmann-De Ridder, T. Gehrmann, E. Glover, and J. Pires, Phys. Rev. Lett. 110, 162003 (2013).

[24] A. Martin, W. Stirling, R. Thorne, and G. Watt, Eur. Phys. J. C 63, 189 (2009).

[25] R. D. Ball et al., Nucl. Phys. B867, 244 (2013).

[26] S. Alekhin, J. Bluemlein, and S. Moch, Phys. Rev. D 86, 054009 (2012).

[27] P. Jimenez-Delgado and E. Reya, Phys. Rev. D 79, 074023 (2009).

[28] The H1 and ZEUS Collaborations, Report No. H1prelim11-042, ZEUS-prel-11-002.

[29] https://www.desy.de/h1zeus/combined_results/index.php? do=proton_structure.

[30] M. Guzzi, P. M. Nadolsky, H.-L. Lai, and C.-P. Yuan, Phys. Rev. D 86, 053005 (2012).

[31] P. Nadolsky et al., arXiv:1206.3321.

[32] H. L. Lai, J. Huston, S. Kuhlmann, F. Olness, J. Owens, D. Soper, W.-K. Tung, and H. Weerts, Phys. Rev. D 55, 1280 (1997).

[33] J. Huston, E. Kovacs, S. Kuhlmann, H. Lai, J. Owens, D. Soper, and W.-K. Tung, Phys. Rev. Lett. 77, 444 (1996).

[34] http://www.cteq.org.

[35] https://hapdf.hepforge.org/.

[36] H.-L. Lai, J. Huston, Z. Li, P. Nadolsky, J. Pumplin, D. Stump, and C.-P. Yuan, Phys. Rev. D 82, 054021 (2010).

[37] S. Kretzer, H.-L. Lai, F. Olness, and W.-K. Tung, Phys. Rev. D 69, 114005 (2004).

[38] R. Thorne and R. Roberts, Phys. Rev. D 57, 6871 (1998).

[39] R. Thorne and R. Roberts, Phys. Lett. B 421, 303 (1998).

[40] M. Cacciari, M. Greco, and P. Nason, J. High Energy Phys. 05 (1998) 007.

[41] S. Forte, E. Laenen, P. Nason, and J. Rojo, Nucl. Phys. B834, 116 (2010).

[42] M. Buza, Y. Matiounine, J. Smith, and W. van Neerven, Eur. Phys. J. C 1, 301 (1998).
[43] A. Chuvakin, J. Smith, and W. van Neerven, Phys. Rev. D 61, 096004 (2000)

[44] I. Bierenbaum, J. Bluemlein, and S. Klein, Phys. Lett. B 672, 401 (2009).

[45] P. M. Nadolsky and W.-K. Tung, Phys. Rev. D 79, 113014 (2009).

[46] T. Stavreva, F. I. Olness, I. Schienbein, T. Ježo, A. Kusina, K. Kovařík, and J. Y. Yu, Phys. Rev. D 85, 114014 (2012).

[47] J. Andersen et al. (SM and NLO Multileg Working Group), arXiv:1003.1241.

[48] G. Aad et al. (ATLAS Collaboration), J. High Energy Phys. 12 (2010) 060.

[49] S. Chatrchyan et al. (CMS Collaboration), J. High Energy Phys. 10 (2011) 132.

[50] G. Watt, J. High Energy Phys. 09 (2011) 069.

[51] R. Thorne, Phys. Rev. D 86, 074017 (2012).

[52] R. D. Ball et al. (NNPDF Collaboration), Phys. Lett. B 723, 330 (2013).

[53] J. Gao, M. Guzzi, and P. M. Nadolsky, Eur. Phys. J. C 73, 2541 (2013).

[54] R. M. Barnett, Phys. Rev. Lett. 36, 1163 (1976).

[55] G. P. Salam and J. Rojo, Comput. Phys. Commun. 180, 120 (2009).

[56] F. Aaron et al. (H1 Collaboration), Eur. Phys. J. C 71, 1769 (2011).

[57] J. Breitweg et al. (ZEUS Collaboration), Eur. Phys. J. C 12, 35 (2000).

[58] S. Chekanov et al. (ZEUS Collaboration), Phys. Rev. D 69 , 012004 (2004).

[59] A. Aktas et al. (H1 Collaboration), Eur. Phys. J. C 45, 23 (2006).

[60] A. Aktas et al. (H1 Collaboration), Eur. Phys. J. C 51, 271 (2007).

[61] H. Abramowicz et al. (H1 Collaboration, ZEUS Collaboration), Eur. Phys. J. C 73, 2311 (2013).

[62] K. Chetyrkin, J. H. Kuhn, and M. Steinhauser, Comput. Phys. Commun. 133, 43 (2000).

[63] S. Alekhin, J. Bluemlein, K. Daum, K. Lipka, and S. Moch, Phys. Lett. B 720, 172 (2013).

[64] J. Beringer et al. (Particle Data Group), Phys. Rev. D 86, 010001 (2012).

[65] J. Pumplin, D. Stump, R. Brock, D. Casey, J. Huston, J. Kalk, H. Lai, and W.-K. Tung, Phys. Rev. D 65, 014013 (2001).

[66] F. Aaron et al. (H1 and ZEUS Collaborations), J. High Energy Phys. 01 (2010) 109.

[67] A. Benvenuti et al. (BCDMS Collaboration), Phys. Lett. B 223, 485 (1989).

[68] A. Benvenuti et al. (BCDMS Collaboration), Phys. Lett. B 237, 592 (1990).

[69] M. Arneodo et al. (New Muon Collaboration), Nucl. Phys. B483, 3 (1997).

[70] J. Berge et al., Z. Phys. C 49, 187 (1991).

[71] U.-K. Yang et al. (CCFR/NuTeV Collaboration), Phys. Rev. Lett. 86, 2742 (2001).

[72] W. Seligman et al., Phys. Rev. Lett. 79, 1213 (1997).

[73] D. A. Mason, Ph.D. thesis, University of Oregon, 2006.

[74] M. Goncharov et al., Phys. Rev. D 64, 112006 (2001).

[75] C. Adloff et al. (H1 Collaboration), Phys. Lett. B 528, 199 (2002). 
[76] A. Aktas et al. (H1 Collaboration), Eur. Phys. J. C 40, 349 (2005).

[77] G. Moreno et al., Phys. Rev. D 43, 2815 (1991).

[78] R. Towell et al. (FNAL E866/NuSea Collaboration), Phys. Rev. D 64, 052002 (2001).

[79] J. Webb et al. (NuSea Collaboration), arXiv:hep-ex/ 0302019.

[80] F. Abe et al. (CDF Collaboration), Phys. Rev. Lett. 77, 2616 (1996).

[81] D. Acosta et al. (CDF Collaboration), Phys. Rev. D 71, 051104 (2005).

[82] V. Abazov et al. (D0 Collaboration), Phys. Rev. Lett. 101, 211801 (2008).

[83] V. Abazov et al. (D0 Collaboration), Phys. Rev. D 77, 011106 (2008).

[84] V. Abazov et al. (D0 Collaboration), Phys. Lett. B 658, 112 (2008).

[85] T. A. Aaltonen et al. (CDF Collaboration), Phys. Lett. B 692, 232 (2010).

[86] T. Aaltonen et al. (CDF Collaboration), Phys. Rev. D 78, 052006 (2008).

[87] V. Abazov et al. (D0 Collaboration), Phys. Rev. Lett. 101, 062001 (2008).

[88] D. Stump, J. Pumplin, R. Brock, D. Casey, J. Huston, J. Kalk, H. Lai, and W.-K. Tung, Phys. Rev. D 65, 014012 (2001).

[89] P. M. Nadolsky and Z. Sullivan, eConf C010630, P510 (2001).

[90] R. A. Fisher, Statistical Methods for Research Workers (Oliver and Boyd, Edinburgh, 1925), Chap. 4; an Internet version of the 1st ed. is available at http://psychclassics .yorku.ca/Fisher/Methods/.

[91] T. Lewis, Australian Journal of statistics 30A, 160 (1988).

[92] G. D’Agostini, Report No. CERN-99-03, CERN-YELLOW-99-03, 1999, Sec. 6.3.

[93] G. D’Agostini, Nucl. Instrum. Methods Phys. Res., Sect. A 346, 306 (1994).

[94] R. D. Ball et al., J. High Energy Phys. 04 (2013) 125.

[95] R. D. Ball, L. Debbio, S. Forte, A. Guffanti, J. I. Latorre, J. Rojo, and M. Ubiali (NNPDF Collaboration), J. High Energy Phys. 05 (2010) 075.

[96] S. Alekhin, J. Bluemlein, and S. Moch, Eur. Phys. J. C 71, 1723 (2011).

[97] R. Thorne and G. Watt, J. High Energy Phys. 08 (2011) 100.

[98] R. D. Ball et al. (NNPDF Collaboration), Phys. Lett. B 704, 36 (2011).

[99] S. D. Ellis, Z. Kunszt, and D. E. Soper, Phys. Rev. Lett. 69, 1496 (1992).

[100] J. Gao, Z. Liang, D. E. Soper, H.-L. Lai, P. M. Nadolsky, and C.-P. Yuan, Comput. Phys. Commun. 184, 1626 (2013).

[101] Z. Nagy, Phys. Rev. D 68, 094002 (2003).

[102] T. Kluge, K. Rabbertz, and M. Wobisch, arXiv:hep-ph/ 0609285.

[103] http://projects.hepforge.org/fastnlo/form/index.html.

[104] M. Wobisch, D. Britzger, T. Kluge, K. Rabbertz, and F. Stober (FastNLO Collaboration), arXiv:1109.1310.

[105] T. Carli, D. Clements, A. Cooper-Sarkar, C. Gwenlan, G. P. Salam, F. Siegert, P. Starovoitov, and M. Sutton, Eur. Phys. J. C 66, 503 (2010).

[106] N. Kidonakis and J. Owens, Phys. Rev. D 63, 054019 (2001).
[107] M. Cacciari, M. Czakon, M. Mangano, A. Mitov, and P. Nason, Phys. Lett. B 710, 612 (2012).

[108] M. Czakon, P. Fiedler, and A. Mitov, Phys. Rev. Lett. 110, 252004 (2013).

[109] R. Gavin, Y. Li, F. Petriello, and S. Quackenbush, Comput. Phys. Commun. 182, 2388 (2011).

[110] R. Gavin, Y. Li, F. Petriello, and S. Quackenbush, Comput. Phys. Commun. 184, 208 (2013).

[111] C. Balazs and C.-P. Yuan, Phys. Rev. D 56, 5558 (1997).

[112] F. Landry, R. Brock, P. M. Nadolsky, and C.-P. Yuan, Phys. Rev. D 67, 073016 (2003).

[113] M. Guzzi and P. M. Nadolsky, Int. J. Mod. Phys. Conf. Ser. 20, 274 (2012).

[114] P. B. Arnold and M. H. Reno, Nucl. Phys. B319, 37 (1989).

[115] P. B. Arnold, R. K. Ellis, and M. Reno, Phys. Rev. D 40, 912 (1989).

[116] R. Hamberg, W. van Neerven, and T. Matsuura, Nucl. Phys. B359, 343 (1991).

[117] A. Cafarella, C. Coriano, and M. Guzzi, J. High Energy Phys. 08 (2007) 030.

[118] A. Cafarella, C. Coriano, and M. Guzzi, Comput. Phys. Commun. 179, 665 (2008).

[119] M. Czakon and A. Mitov, arXiv:1112.5675.

[120] P. Baernreuther, M. Czakon, and A. Mitov, Phys. Rev. Lett. 109, 132001 (2012).

[121] S. Catani and M. Grazzini, Phys. Rev. Lett. 98, 222002 (2007).

[122] M. Grazzini, J. High Energy Phys. 02 (2008) 043.

[123] S. Dittmaier et al. (LHC Higgs Cross Section Working Group), arXiv:1101.0593.

[124] R. V. Harlander and W. B. Kilgore, Phys. Rev. D 68, 013001 (2003).

[125] G. Aad et al. (ATLAS Collaboration), Phys. Rev. D 85, 072004 (2012).

[126] CMS Collaboration, Report No. CMS-PAS-SMP-12-011.

[127] The Tevatron Electroweak Working Group, D0 Note No. 6363, http://tevewwg.fnal.gov/top/tev_ttbarxsec _summary.pdf.

[128] ATLAS Collaboration, Reports No. ATLAS-CONF-2012024 and No. ATLAS-COM-CONF-2012-009.

[129] G. Aad et al.(ATLAS Collaboration), Phys. Lett. B 717, 89 (2012).

[130] ATLAS Collaboration, Reports No. ATLAS-CONF-2012131 and No. ATLAS-COM-CONF-2012-056.

[131] G. Aad et al. (ATLAS Collaboration), Eur. Phys. J. C 73, 2328 (2013).

[132] ATLAS Collaboration, Report No. ATLAS-CONF-2012-149.

[133] S. Chatrchyan et al. (CMS Collaboration), Phys. Lett. B 720, 83 (2013).

[134] S. Chatrchyan et al. (CMS Collaboration), Phys. Rev. Lett. 109, 111806 (2012).

[135] R. Aaij et al. (LHC-B Collaboration), J. High Energy Phys. 06 (2012) 058.

[136] K. Melnikov and F. Petriello, Phys. Rev. D 74, 114017 (2006).

[137] S. Catani, L. Cieri, G. Ferrera, D. de Florian, and M. Grazzini, Phys. Rev. Lett. 103, 082001 (2009).

[138] M. Guzzi et al., arXiv:1101.0561.

[139] CMS Collaboration, Report No. CMS-PAS-SMP-12-021.

[140] G. Aad et al. (ATLAS Collaboration), Phys. Rev. Lett. 109, 012001 (2012). 